\title{
Optical studies of semiconductor perovskite nanocrystals for classical optoelectronic applications and quantum information technologies: a review
}

\author{
Zengle Cao, ${ }^{\text {a, }}$ Fengrui Hu, ${ }^{\text {a, }}$ Chunfeng Zhang, ${ }^{a}$ Shining Zhu, ${ }^{a}$ Min Xiao, ${ }^{a, b}$ and Xiaoyong Wang ${ }^{a, *}$ \\ ${ }^{a}$ Nanjing University, College of Engineering and Applied Sciences, School of Physics, Collaborative Innovation Center of Advanced Microstructures, \\ National Laboratory of Solid State Microstructures, Nanjing, China \\ 'University of Arkansas, Department of Physics, Fayetteville, Arkansas, United States
}

\begin{abstract}
Semiconductor perovskite films are now being widely investigated as light harvesters in solar cells with ever-increasing power conversion efficiencies, which have motivated the fabrication of other optoelectronic devices, such as light-emitting diodes, lasers, and photodetectors. Their superior material and optical properties are shared by the counterpart colloidal nanocrystals (NCs), with the additional advantage of quantum confinement that can yield size-dependent optical emission ranging from the near-UV to near-infrared wavelengths. So far, intensive research efforts have been devoted to the optical characterization of perovskite NC ensembles, revealing not only fundamental exciton relaxation and recombination dynamics but also lowthreshold amplified spontaneous emission and novel superfluorescence effects. Meanwhile, the application of single-particle spectroscopy techniques to perovskite NCs has helped to resolve a variety of optical properties for which there are few equivalents in traditional colloidal NCs, mainly including nonblinking photoluminescence, suppressed spectral diffusion, stable exciton fine structures, and coherent singlephoton emission. While the main purpose of ensemble optical studies is to guide the smooth development of perovskite NCs in classical optoelectronic applications, the rich observations from single-particle optical studies mark the emergence of a potential platform that can be exploited for quantum information technologies.

Keywords: perovskite nanocrystals; optical studies; single-particle spectroscopy; transient absorption; optoelectronic applications; quantum information technologies.
\end{abstract}

Received Jun. 12, 2020; revised manuscript received Aug. 12, 2020; accepted for publication Aug. 25, 2020; published online Sep. 21, 2020.

(c) The Authors. Published by SPIE and CLP under a Creative Commons Attribution 4.0 Unported License. Distribution or reproduction of this work in whole or in part requires full attribution of the original publication, including its DOI.

[DOI: 10.1117/1.AP.2.5.054001]

Perovskite structures are named after the Russian mineralogist Lev A. Perovski to initially describe the $\mathrm{CaTiO}_{3}$ material that was discovered in 1839 by the German mineralogist Gustav Rose from the Russian Ural mountains. ${ }^{1,2}$ Nowadays, the term "perovskite" has been widely extended to any material adopting the $\mathrm{CaTiO}_{3}$-like $\mathrm{ABX}_{3}$ structure, where $\mathrm{A}$ is an organic or alkali-metal cation [e.g., $\mathrm{CH}_{3} \mathrm{NH}_{3}{ }^{+}\left(\mathrm{MA}^{+}\right), \mathrm{HC}\left(\mathrm{NH}_{2}\right)_{2}{ }^{+}\left(\mathrm{FA}^{+}\right)$,

*Address all correspondence to Xiaoyong Wang, E-mail: wxiaoyong@nju.edu.cn †These authors contributed equally to this work. or $\mathrm{Cs}^{+}$], $\mathrm{B}$ is a bivalent cation (e.g., $\mathrm{Pb}^{2+}$ or $\mathrm{Sn}^{2+}$ ), and $\mathrm{X}$ is a monovalent anion (e.g., $\mathrm{Cl}^{-}, \mathrm{Br}^{-}$, or $\mathrm{I}^{-}$). ${ }^{3,4}$ As schematically shown in Fig. 1(a) for a typical perovskite structure, the A cation occupies the central position of a unit cell, with the $\mathrm{BX}_{6}$ octohedra being evenly located at the eight surrounding corners. ${ }^{3}$

Although the first scientific report of perovskite materials can be dated back to as early as $1893,{ }^{6}$ they started drawing great research attention only around 2009 after being tentatively used as the sensitizer components for solar cells. ${ }^{7}$ Owing mainly to the large absorption cross sections and excellent charge transport properties, ${ }^{8-10}$ the power conversion efficiencies of solar 
(a)
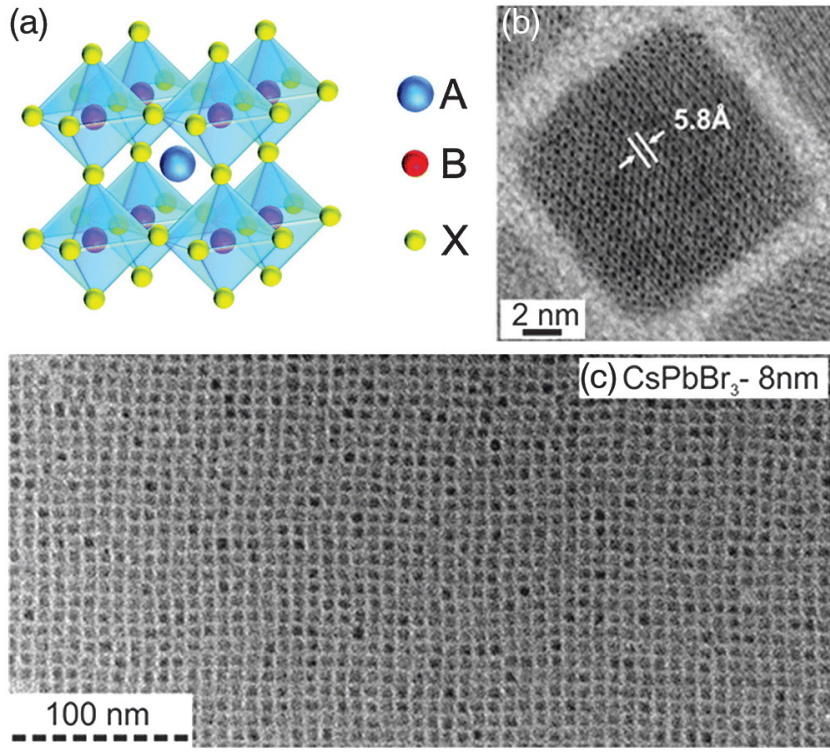

Fig. 1 (a) Schematic of the perovskite $A B X_{3}$ structure, where $A$ is an organic or alkali-metal cation, $B$ is a bivalent cation, and $X$ is a monovalent anion. (b), (c) Typical TEM images of perovskite $\mathrm{CsPbBr}_{3}$ NCs. (a) Reproduced with permission from Ref. 3, courtesy of the Royal Society of Chemistry. (b), (c) Reproduced with permission from Ref. 5, courtesy of the American Chemical Society (ACS).

cells based on the perovskite films have since been continuously elevated to reach more than $25 \%$ at the current stage. ${ }^{11-15}$ Meanwhile, the perovskite films are behaving equally well in light-emitting diode and laser applications, ${ }^{16-19}$ which take advantage of their widely tunable emission wavelengths and high color purities. However, the small binding energy that prevents efficient exciton formation, together with the defect abundance that induces nonradiative exciton decays, normally results in very poor fluorescent quantum yields (QYs) for the perovskite films. ${ }^{20}$

The superior performances of bulk perovskite films in optoelectronic devices have driven the appearance of their counterpart nanocrystals (NCs), ${ }^{21}$ nanowires, ${ }^{22}$ and nanoplatelets, ${ }^{23}$ which are featured with facile synthesis, low cost, and ease in compositional and structural controls. The as-synthesized colloidal perovskite nanomaterials possess a larger exciton binding energy $^{5}$ and a better defect tolerance, ${ }^{24}$ which lead to higher fluorescent QYs especially in the NC case. Borrowing the ligandassisted chemical approach in preparing traditional colloidal NCs, Schmidt et al. ${ }^{21}$ made the first attempt in 2014 to synthesize perovskite $\mathrm{NCs}$ in the hybrid form of organic-inorganic $\mathrm{MAPbBr}_{3}$. From the transmission electron microscopy (TEM) images shown in Figs. 1(b) and 1(c), Protesescu et al. ${ }^{5}$ then developed the hot injection method to synthesize all-inorganic perovskite $\mathrm{CsPbX}_{3}$ NCs. This was followed by the synthesis of organic-inorganic $\mathrm{MAPbX}_{3} \mathrm{NCs}$ from Zhang et al., ${ }^{25}$ using the ligand-assisted reprecipitation (LARP) method under the ambient environment. Hot injection and LARP have since become the two main methods for the synthesis of perovskite nanomaterials whose compositions, sizes, and shapes can be flexibly adjusted by manipulating the precursor salts, ligand types, and reaction conditions. ${ }^{26-28}$ In addition, various protocols have been developed for postsynthesis treatments of perovskite nanomaterials so that their compositional and structural parameters can be further tuned through ion exchanges and surface exfoliations. ${ }^{29-32}$ By means of the rich synthesis and postsynthesis procedures described above, the optical emission of perovskite NCs can now range from the near-UV to near-infrared wavelengths, ${ }^{5,25,33}$ with a fluorescent QY close to $100 \%$ being easily achieved. ${ }^{34-36}$ It is thus not surprising that the last several years have seen tremendous applications of perovskite NCs in solar cell, ${ }^{37-40}$ light-emitting diode, ${ }^{41-47}$ laser, ${ }^{48-50}$ photodetector, ${ }^{51-54}$ and $\mathrm{x}$-ray imaging ${ }^{55,56}$ devices that used to rely heavily on traditional colloidal NCs of metal chalcogenides and III-V compounds. ${ }^{57-59}$

Due to the quantum confinement effect in semiconductor nanostructures, the electronic wave functions are strongly modified to yield discrete energy levels for the band-edge charge carriers. ${ }^{60,61}$ With the concomitant developments of singleparticle spectroscopy ${ }^{62}$ and single-photon detection ${ }^{63}$ techniques, various interesting optical phenomena have been discovered in traditional colloidal NCs, such as fluorescent blinking, ${ }^{64,65}$ singlephoton emission, ${ }^{66}$ exciton fine-structure splitting, ${ }^{67}$ and quantumconfined Stark effect. ${ }^{68,69}$ As a new member just stepping into the colloidal NC family, the perovskite NCs should be subjected to intensive single-particle optical characterization to judge whether they will behave differently in any of the abovementioned photophysical processes. Meanwhile, it is highly expected that new developments should be brought about in the optical studies of single perovskite NCs, especially under the contexts of strong spin-orbit coupling,${ }^{70}$ large absorption cross section, ${ }^{71}$ and fast carrier migration ${ }^{72}$ that have been widely discussed in their counterpart bulk films.

In this review, we focus on the optical studies of perovskite $\mathrm{NCs}$ with the quantum confinement in all three dimensions since those of perovskite nanowires and nanoplatelets are still at the infancy stage due to their unsatisfactory material and optical stabilities. The optical studies of perovskite NC ensembles are valuable in guiding their efficient applications in classical optoelectronic devices, the current status of which has been detailed in several recent reviews. ${ }^{73,74}$ At the single-particle level, the perovskite NCs are associated with unique single-photon emission characteristics and exciton fine structures, which have outlined a prospective route toward their potential usage in quantum information technologies.

\section{Ensemble Optical Properties}

The optoelectronic device applications of semiconductor perovskite NCs are based on their high-density ensemble films, whose optical characterization can provide valuable information on the optimization of relevant operation parameters. The absorption and emission spectra of perovskite $\mathrm{NC}$ ensembles can be routinely measured right after synthesis, which is extremely helpful in working out suitable ion compositions and crystal structures for boosting the fluorescent QYs and enhancing the chemical/optical stabilities. The time-resolved photoluminescence (PL) and transient absorption (TA) techniques can then be employed to probe the carrier relaxation and recombination dynamics as well as the charge transport and extraction processes. Under both one- and multi-photon excitations of ultrafast laser pulses, amplified spontaneous emission (ASE) has been demonstrated in perovskite $\mathrm{NC}$ ensembles with the reported low thresholds being beneficial for the design of highly efficient laser devices. When self-organized into highly ordered superlattices, 
the ensemble perovskite NCs can interact with each other coherently via a common light field, the manipulation of which will promote the realizations of superfluorescent lasers and entangled multiphoton light sources.

\subsection{Fundamental Optical Properties}

As can be seen from the optical images in Fig. 2(a), the solution $\mathrm{CsPbX}_{3} \mathrm{NCs}$ with varying halide compositions can demonstrate different emission colors upon UV-light illumination, which is also reflected in the corresponding PL spectra measured in Fig. 2(b). This composition dependence of bandgap energy is further revealed in Fig. 2(c), where the band-edge absorption and $\mathrm{PL}$ peaks of $\mathrm{CsPbX}_{3} \mathrm{NCs}$ can be tuned within 400 to $700 \mathrm{~nm}$ to cover almost the entire visible-wavelength range. With the subsequent synthesis of $\mathrm{CsSnI}_{3}$ and $\mathrm{FAPbI}_{3}$, the bandgap energy of perovskite NCs can be significantly reduced to arrive at the near-infrared wavelength of $\sim 900 \mathrm{~nm}^{33,75}$ Once the material compositions are fixed, the bandgap energies of perovskite NCs can be additionally tuned by changing the sizes [see Fig. 2(d)], which are normally close to the exciton Bohr diameters to render a relatively weak quantum-confinement effect. ${ }^{5}$ With the changing compositions and sizes in different batches of perovskite NCs, the recombination lifetimes of bandedge excitons can vary from 1 to $70 \mathrm{~ns}$ according to the timeresolved PL measurements performed at room temperature. ${ }^{5,25,75}$

For the practical applications in commercial optoelectronic devices, the perovskite NCs have to be assembled into high-density films where their mutual interactions are unavoidably present. Based on the time-integrated and -resolved PL measurements, de Weerd et al. ${ }^{76}$ confirmed the existence of energy transfer from smaller- to larger-sized perovskite NCs, which could be positively employed for directional funneling of the photoexcited energies. Zhang et al. ${ }^{77}$ discovered that the iodine ions could migrate among mixed-halide $\mathrm{CsPbBr}_{1.2} \mathrm{I}_{1.8}$ NCs contained in a high-density film, leading to a blueshift in the PL peak upon light illumination that would recover back to the red side in the dark.
Relevant to the thermal effect in operating optoelectronic devices, the temperature-dependent optical properties of perovskite NCs also deserved to be thoroughly investigated. With the increasing temperatures, most of the studied perovskite NCs demonstrated a blueshift in the PL peaks with broadened linewidths, as a joint effect of lattice expansion and exciton-phonon coupling. ${ }^{78-81}$ In a very rare occasion, Wei et al. ${ }^{79}$ reported an anomalous redshift observed at $220 \mathrm{~K}$ in the PL peaks of $\mathrm{CsPbBr}_{3} \mathrm{NC}$ ensembles, which was attributed to the electronphonon coupling effect. When the temperature was further increased to be above $300 \mathrm{~K}$, Li et al. ${ }^{80}$ found out that the inorganic perovskite NCs could be thermally deteriorated to cause an irreversible quench in the PL intensities. The temperaturedependent optical properties are also strongly dependent on the existence of defect sites, which have been shown by Ma et al. ${ }^{81}$ to cause a phase transition from cubic to orthorhombic crystal structures upon cooling all-inorganic perovskite NCs from room temperature.

\subsection{Transient Absorption Measurements}

As schematically shown in Fig. 3(a), the charge carriers would undergo complex dynamic processes once being excited into the higher-lying excited states of perovskite NCs, mainly including thermal relaxation, state filling, Auger interaction, and radiative recombination ${ }^{82}$ For the time-resolved PL techniques employed in the last section, the best resolution that can be achieved with state-of-the-art photon detectors is at the scale of tens of picoseconds, which is adequate only for measuring the slow recombination lifetime of band-edge excitons. In contrast, the ultrafast TA technique can possess a subpicosecond time resolution limited mainly by the laser pulse width, making it a powerful tool in characterizing the versatile dynamic processes depicted in Fig. 3(a). Right after excitation of the pump laser, hot excitons are created in perovskite NCs, and their Coulomb interactions are capable of inducing a redshift in the bandgap energy that can be detected by the probe laser. ${ }^{83}$ This will cause a reduced absorption or exciton bleach in the bandgap energy and a photo-induced absorption peak at smaller energies, as
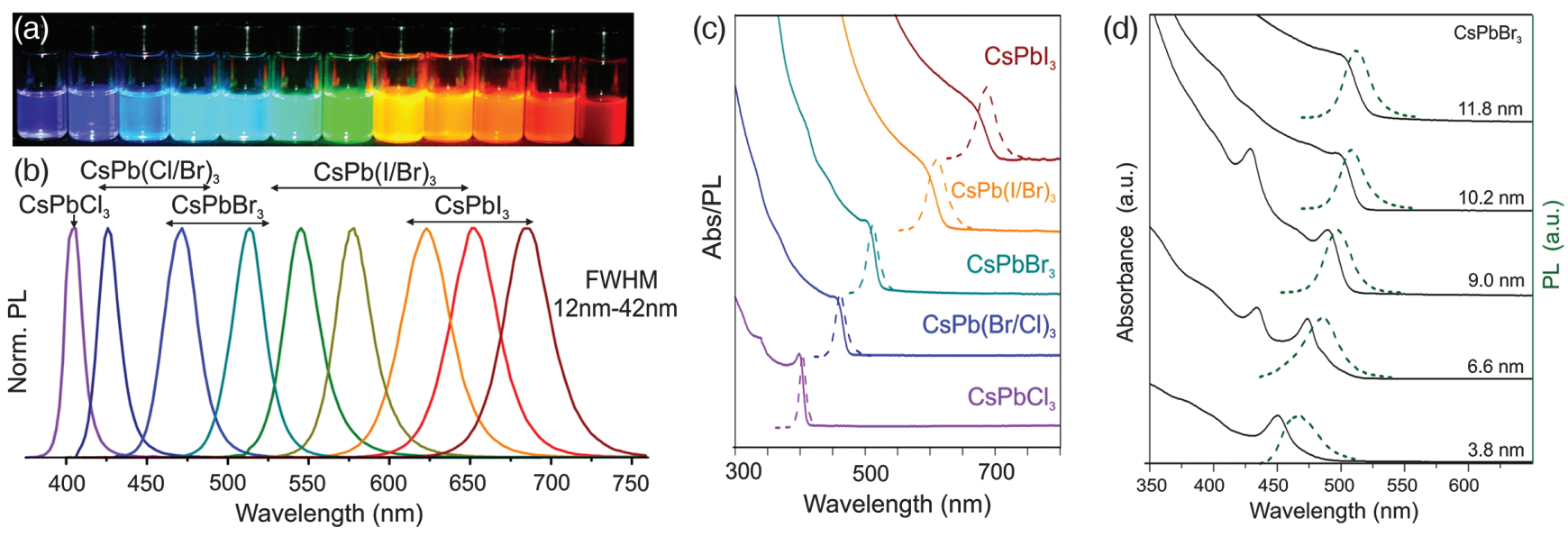

Fig. 2 (a) Optical images of solution $\mathrm{CsPbX}_{3} \mathrm{NCs}$ excited by a UV lamp and (b) the corresponding $\mathrm{PL}$ spectra. (c) Absorption and PL spectra of $\mathrm{CsPbX}_{3}$ NCs with different halide compositions. (d) Absorption and PL spectra of $\mathrm{CsPbBr}_{3} \mathrm{NCs}$ with different sizes. Reproduced with permission from Ref. 5, courtesy of ACS. 
(a)

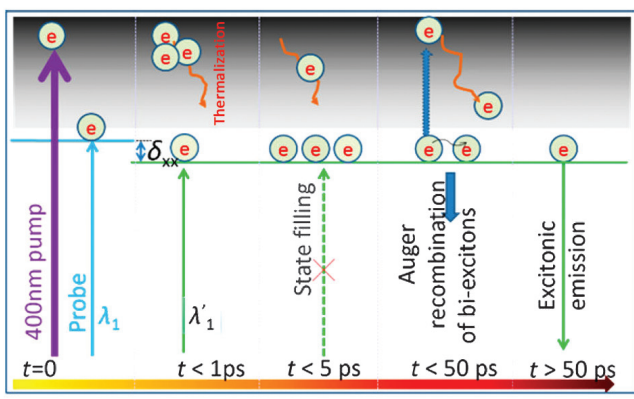

(b)

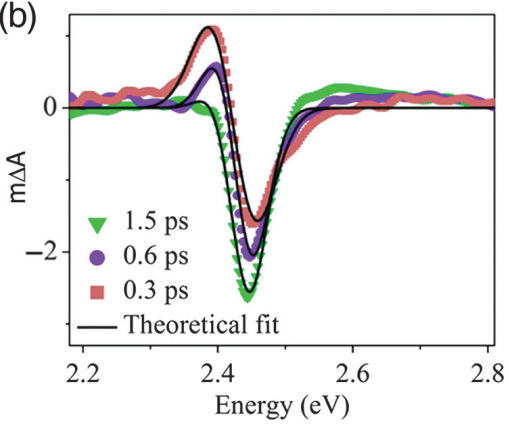

Fig. 3 (a) Schematic for the photoexcited carrier dynamics in perovskite NCs. (b) Typical TA spectra of $\mathrm{CsPbBr}_{3} \mathrm{NCs}$ measured at different time delays between the pump and probe laser pulses. Reproduced with permission from Ref. 82, courtesy of ACS.

shown in Fig. 3(b) from several typical TA spectra measured for perovskite $\mathrm{CsPbr}_{3} \mathrm{NCs}^{82}$ Accompanied by a slow buildup of the exciton bleach signal, the photo-induced absorption disappears within 1 ps after laser excitation, signifying almost complete thermal relaxation of hot excitons into the band-edge states at this time scale. ${ }^{83,84}$ Under high-power laser excitation, the many excitons dwelling at the band-edge states can interact with each other through nonradiative Auger recombination, which will produce a fast decay of the exciton bleach signal within 10 to 100 ps. $^{82,85,86}$ Interestingly, it was revealed by Mondal et $\mathrm{al}^{87}$ that the many hot excitons created in perovskite NCs by high laser powers might suffer from the phonon bottleneck effect, where their relaxation process could be significantly impeded due to the lack of available phonons for dissipating the extra thermal energy.

After going through the above ultrafast dynamic processes, the perovskite NCs are left with band-edge excitons whose radiative lifetime can be conveniently measured by the timeresolved PL technique. However, the TA measurement has to be invoked again whenever there exists nonradiative decay channels for band-edge excitons, as exemplified by Luo et al ${ }^{88}$ in $\mathrm{MAPbBr}_{3} \mathrm{NCs}$ that the defect traps from surface ligands could capture the photoexcited charge carriers within 6 to $50 \mathrm{ps}$. The powerfulness of the TA technique also lies in the fact that it can always pick up observations in the ultrafast studies of perovskite NCs, which are not depicted in the schematic shown in Fig. 3(a) and even not expected before people set out to do the experiments. For example, Rossi et al. ${ }^{89}$ observed anomalous photoinduced absorption in $\mathrm{CsPbr}_{3} \mathrm{NC}$ ensembles with an energy higher than that of the exciton bleach peak, which they attributed to the breaking of forbidden exciton transitions through the formation of carrier-lattice coupled polarons.

Besides characterizing the intrinsic exciton relaxation and recombination dynamics of perovskite NCs, the TA technique has proven to be extremely helpful in figuring out efficient carrier migration and transfer schemes for their applications in optoelectronic devices. The charge migration rate and diffusion length of $\mathrm{CsPBrr}_{3} \mathrm{NC}$ films were estimated by Yettapu et al. ${ }^{90}$ to be $\sim 4500 \mathrm{~cm}^{2} \mathrm{~V}^{-1} \mathrm{~s}^{-1}$ and $\sim 9.2 \mu \mathrm{m}$, respectively, which are on a par with or even surmount those values possessed by their counterpart bulk single crystals and films. ${ }^{91-95}$ Meanwhile, it takes 0.1 to $100 \mathrm{ps}$ for the photoexcited electrons or holes to be transferred from perovskite NCs to the surrounding organic molecules, ${ }^{96-99}$ resulting in a charge-separation state that can persist even after several microseconds. ${ }^{100}$ While this charge-separation state is beneficial for realizing efficient charge extraction in photovoltaics, it can be further extended to the photocatalytic and light-harvesting applications according to a recent study by Dana et al. ${ }^{101}$ on the charge transfer process between perovskite $\mathrm{CsPbr}_{3}$ and traditional CdSe NCs. In addition to the chargetransfer interaction, triplet energy transfer across the interface formed by perovskite NCs and organic molecules has been discovered, ${ }^{102,103}$ making this hybrid inorganic-organic system a potential candidate for being used as photon upconverters. ${ }^{104}$

\subsection{Amplified Spontaneous Emission}

The time resolution in the above TA measurements relies critically on the pulse widths of the ultrafast laser sources, which are also indispensable in triggering ASE in perovskite NC ensembles, especially at the femtosecond and high-energy regimes. As shown in Figs. 4(a) and 4(b), Yakunin et al ${ }^{48}$ reported the ASE effect of $\mathrm{CsPbBr}_{3} \mathrm{NCs}$ right after they were first synthesized in 2015 , with the threshold being as low as $\sim 5 \mu \mathrm{J} \mathrm{cm}^{-2}$. The ASE wavelengths could be easily tuned by changing the halide compositions [Fig. 4(c)] and, besides the random lasing observed in solid films, the microcavity lasing was also achieved in this work by coupling perovskite NCs with the whispering gallery modes of silicon microspheres. While the easy demonstration of ASE and lasing behaviors in perovskite NCs are related to their large absorption cross sections and high fluorescent QYs, the highly efficient nonlinear absorption properties can further help to implement the ASE processes under the two- and multiple-photon pumping conditions. ${ }^{106-108}$ After the ASE of $\mathrm{CsPbBr}_{3} \mathrm{NCs}$ was reported by Pan et al. with a threshold of $12 \mathrm{~mJ} \mathrm{~cm}^{-2},{ }^{106}$ their laser operations were soon realized with a threshold of only $0.9 \mathrm{~mJ} \mathrm{~cm}^{-2},{ }^{109}$ both with two-photon excitations to take advantage of the large absorption cross section of $2.7 \times 10^{6} \mathrm{GM}\left(1 \mathrm{GM}=10^{-50} \mathrm{~cm}^{4} \mathrm{~s} /\right.$ photon $)$ for this perovskite nanomaterial. ${ }^{109}$ Meanwhile, great research attention has also been paid to the three-photon pumped ASE, with a low threshold of $\sim 5.2 \mathrm{~mJ} \mathrm{~cm}^{-2}$ being reported for $\mathrm{CsPbBr}_{3} \mathrm{NCs}^{107}$

The population inversion and optical gain in perovskite NCs are strongly dependent on the existence of biexcitons normally dissipated within $100 \mathrm{ps}$ through the nonradiative Auger recombination process, ${ }^{83}$ which sets a stringent limit on the ASE establishment time and hence the corresponding threshold. In traditional colloidal NCs of metal chalcogenides, it was observed that intentional charging could lower the ASE threshold,${ }^{110,111}$ whose working principle is schematically shown in 

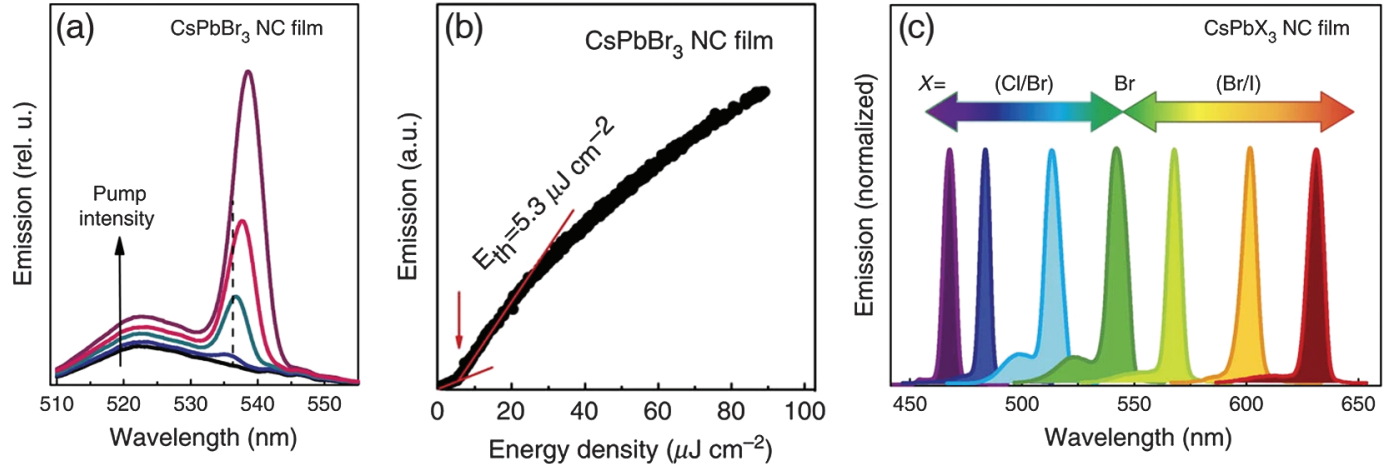

(d) Biexciton gain in QDs

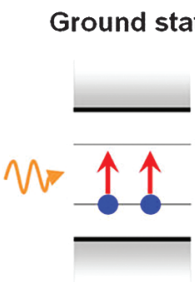

Abs. > SE

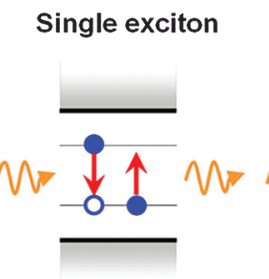

Abs. $=$ SE

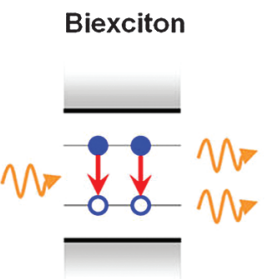

Abs. $<$ SE
Trion gain in QDs

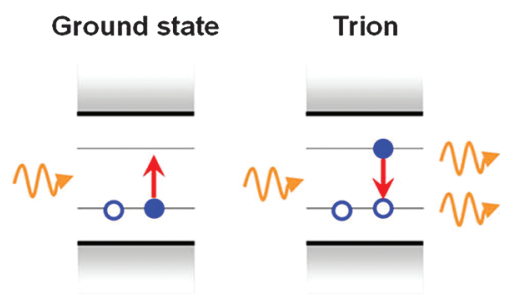

Abs. > SE

Abs. < SE

Fig. 4 (a) PL spectra measured as a function of the pump fluence for a solid film of $\mathrm{CsPbBr}_{3}$ NCs and (b) the corresponding threshold behavior for the ASE-band PL intensity. (c) Spectral tunability of the ASE band by means of compositional modulation. (d) Comparison between the mechanisms of biexciton gain and trion gain in neutral (left) and singly charged (right) colloidal NCs. (a)-(c) Reproduced with permission from Ref. 48, courtesy of Macmillan Publishers Limited. (d) Reproduced with permission from Ref. 105, courtesy of ACS.

Fig. 4(d). ${ }^{105}$ Using a mixture of $\mathrm{PbBr}_{2}$, oleic acid, and oleylamine in 2018, Wang et al. ${ }^{105}$ modified the surface of $\mathrm{CsPbBr}_{3}$ $\mathrm{NCs}$ to obtain long-lived charged excitons and to achieve an ultra-low threshold of $1.2 \mu \mathrm{J} \mathrm{cm}^{-2}$ for the one-photon pumped ASE process. It should be noted that the chemical stability is one of the main concerns in the above ASE studies of perovskite NCs with high laser pulse energies, which is now being actively addressed by the surface passivation ${ }^{105}$ and inorganic coating ${ }^{112}$ methods.

\subsection{Superfluorescence}

In the above ASE processes, coherent interactions are not expected among the emission dipoles of individual perovskite NCs. However, if an ensemble of optical emitters can interact coherently via a common light field, their correlated emission dipoles would give rise to short and intense bursts of light [see Fig. 5(a)]. ${ }^{115}$ This so-called superfluorescence effect was recently observed by Rainò et al. in three-dimensional micrometer-sized superlattices composed of self-organized, longrange-ordered $\mathrm{CsPbBr}_{3} \mathrm{NCs}$ [see Fig. 5(b)], ${ }^{113}$ implying their uniqueness in homogeneous size distribution and low optical decoherence that are hardly possessed by any other colloidal NC systems. The novel optical features of superfluorescence such as redshifted energy [Fig. 5(c)], much shortened lifetime, bunched photon emission [Fig. 5(d)], and Burnham-Chiao ringing have opened exclusive pathways for the cooperative applications of perovskite $\mathrm{NCs}$ in multiphoton quantum light sources.
Meanwhile, it might be feasible to embed perovskite NCs inside an optical cavity for the construction of superfluorescent lasers already achieved in atomic systems, ${ }^{116,117}$ wherein the cooperative exciton recombination can result in an instant consumption of all coherent emission dipoles. In a recent attempt by Zhou et al., ${ }^{114}$ the individual superlattices made of $\mathrm{CsPbBr}_{3} \mathrm{NCs}$ could be assembled again to form the microcavity with a quality factor of $\sim 1800$. The occurrence of superfluorescence lasing could be deduced from the pumping-density dependences of the PL peaks and intensities shown in Figs. 5(e) and 5(f), respectively, and was also reflected in the associated superfluorescent lifetime of 4 ps as compared to that of 29 ps without the cavity enhancement. Overall, the successful observation of the superfluorescence effect and the subsequent demonstration of superfluorescence lasing have started driving perovskite NC ensembles into the quantum-information-processing regime, a goal that is also being currently pursued in their single-particle optical studies.

\section{Single-Particle Optical Properties}

Aside from the optical studies of perovskite NC ensembles for the good use of them in classical optoelectronic applications, the single-particle optical characterization is essential in uncovering intrinsic quantum-light characteristics that are increasingly required for investigating the artificial-atom physics and promoting the potential applications in quantum information technologies. The discrete energy levels resulting from the quantum 
(a)

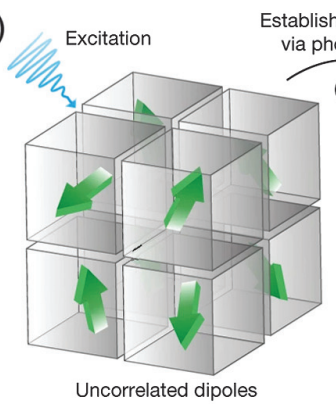
photon field

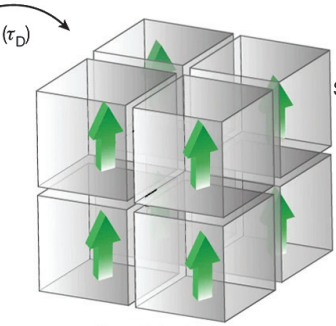

Correlated dipoles (b)

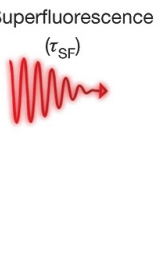

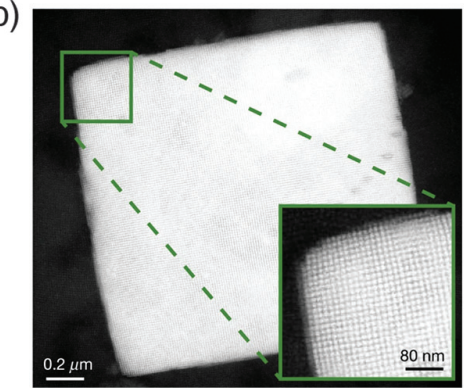

(c)

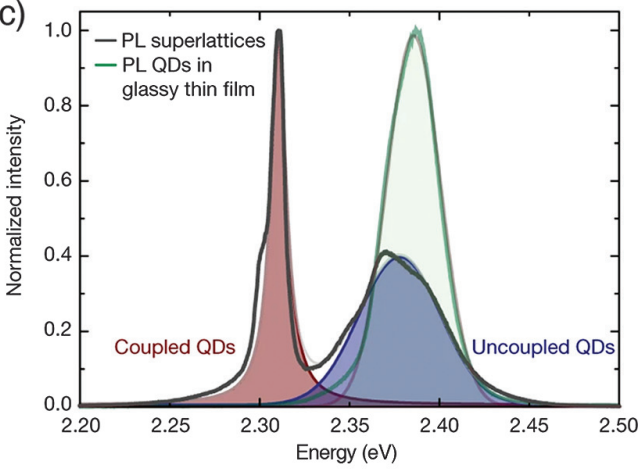

(e)

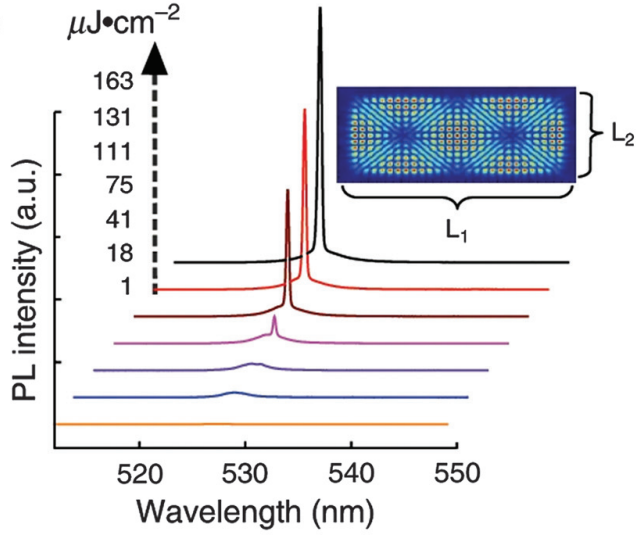

(d)

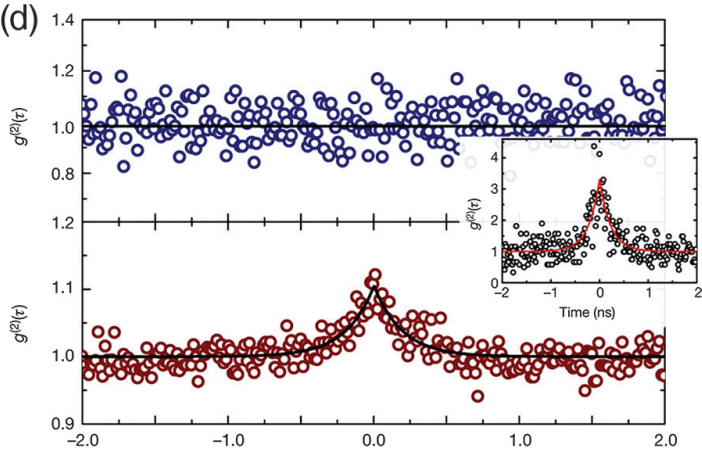

(f)

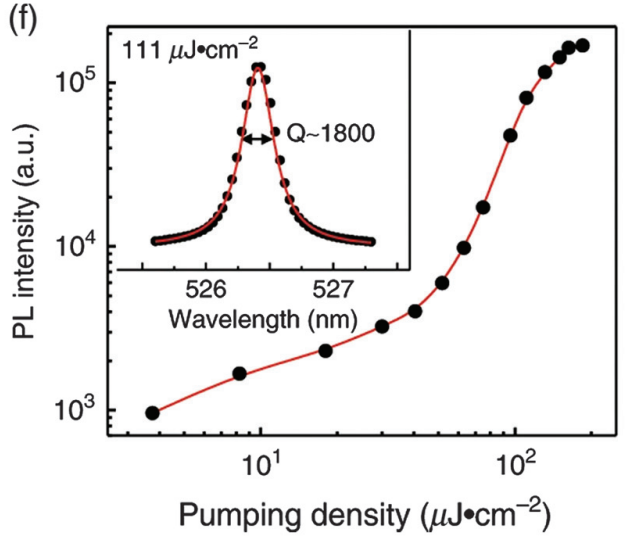

Fig. 5 (a) Schematic for the buildup process of superfluorescence. (b) High-angle annular darkfield scanning TEM image of a single superlattice composed of $\mathrm{CsPbBr}_{3} \mathrm{NCs}$. Inset: A magnified view for part of the superlattice showing individual $\mathrm{CsPbBr}_{3}$ NCs. (c) PL spectra measured for $\mathrm{CsPbBr}_{3} \mathrm{NCs}$ contained in a superlattice and a normal film, respectively. The high- and lowenergy bands are assigned to uncoupled and coupled NCs, respectively. (d) Second-order autocorrelation functions measured for high- (upper graph) and low-energy (lower graph) bands, respectively. Inset: An example of superbunching from a single superlattice of $\mathrm{CsPbBr}_{3} \mathrm{NCs}$. (e) PL spectra measured for $\mathrm{CsPBr}_{3} \mathrm{NCs}$ in a superlattice microcavity showing enhanced superfluorescence. Inset: Simulated field distribution of the whispering gallery mode in this microcavity. (f) PL intensities of the cavity mode measured as a function of the laser pumping intensity. Inset: Lorentz fitting of the cavity mode with a quality factor of $\sim 1800$. (a)-(d) Reproduced with permission from Ref. 113, courtesy of Springer Nature Limited. (e), (f) Reproduced with permission from Ref. 114.

confinement, when coupled with the defect-tolerant feature, can render single-photon emission from a single perovskite $\mathrm{NC}$ with suppressed PL blinking and spectral diffusion effects. This has allowed the discovery of stable exciton fine structures in single perovskite NCs, which could originate from either the electronhole exchange interaction or the Rashba effect. The exciton dephasing time in the emission state of a single perovskite $\mathrm{NC}$ can now be estimated from photon-correlation Fourier spectroscopy (PCFS), while the realization of quantum interference measurement on the absorption state has represented the first example of coherently manipulating the exciton wave functions in a colloidal NC system. Owing to the large absorption cross section, the two-photon excitation approach is expected to play an important role in studying the quantum optical properties of single 
perovskite NCs, especially under the near-resonant and resonant conditions with the greatly attenuated background signals.

\subsection{Single-Photon Emission Characteristics}

The quantum-confinement effect in single colloidal NCs can yield discrete energy levels for the single-photon emission from neutral excitons. ${ }^{66}$ However, it can also reinforce nonradiative Auger recombination of charged excitons. ${ }^{59}$ Thus, even under continuous laser excitation, the PL intensity of a single colloidal NC could switch between the "off" and "on" states due to the fast charging and discharging events, the former of which arises mainly from surface trapping of the photoexcited electrons or holes. ${ }^{64}$ The surface-trapped charge carriers are capable of imposing local electric fields on a single colloidal NC, so that the above fluorescent blinking effect is normally accompanied by spectral diffusion that can significantly broaden the measured PL linewidth. ${ }^{118}$ Due to the existence of fluorescence blinking in a single colloidal NC, the fluorescent QY is greatly jeopardized and the single photons can only be detected intermittently to bring uncertainties in their further manipulations. Over the last several decades, tremendous chemical approaches have been tested in order to overcome this annoying optical deficiency in traditional colloidal NCs by means of suppressing the nonradiative Auger recombination and reducing the surface carrier traps. ${ }^{119-122}$ However, it is still a very challenging goal to eliminate fluorescent blinking and spectral diffusion simultaneously, together with an ultranarrow PL linewidth measured stably at cryogenic temperatures.

Single-photon emission from single perovskite $\mathrm{CsPbX}_{3} \mathrm{NCs}$ was reported by Park et al. ${ }^{123}$ in 2015 under both continuouswave [Fig. 6(a)] and pulsed [Fig. 6(b)] laser excitations, which suffered a lot from the fluorescence blinking effect [Fig. 6(c)].
At the same time, single-photon emission and the associated fluorescence blinking were also reported by Hu et al. ${ }^{125}$ in single $\mathrm{CsPbBr}_{3} \mathrm{NCs}$, which additionally demonstrated several superior optical properties such as a large absorption cross section, a fast radiative lifetime, and a suppressed dark-exciton emission. Sharma et al. ${ }^{126}$ later discovered that the optical and chemical properties of single $\mathrm{CsPbBr}_{3} \mathrm{NCs}$ could be strongly influenced by the surrounding polymers, thus suggesting an alternative way of adjusting their relative energy-level alignments for a full suppression of the fluorescence blinking effect.

Surprisingly, $\mathrm{Hu}$ et al. ${ }^{124}$ later discovered that the fluorescence blinking behavior was completely missing in single $\mathrm{CsPbI}_{3} \mathrm{NCs}$ under low-power laser excitation, as can be seen from the PL intensity time trace plotted in Fig. 6(d). Due to the accompanied suppression of spectral diffusion, a resolutionlimited PL linewidth could be reliably measured for a single $\mathrm{CsPbI}_{3} \mathrm{NC}$ at the cryogenic temperature of $4 \mathrm{~K}$ [see Fig. 6(e)]. The above optical stabilities are more or less possessed by single perovskite NCs with other compositions such as $\mathrm{CsPb}(\mathrm{Cl} / \mathrm{Br})_{3}{ }^{127}$ and $\mathrm{FAPbBr}_{3},{ }^{128}$ signifying the important role played by defect tolerance ${ }^{129}$ in removing the carrier traps for band-edge excitons. The ultranarrow PL linewidths, which are readily available from these single perovskite NCs and robust during the measurement time, have become the decisive factor in resolving their exciton fine structures.

\subsection{Exciton Fine Structures}

According to the density-functional-theory calculations performed on metal-halide perovskites, ${ }^{130,131}$ the valence band maximum comes from a hybridization of the $s$-orbitals of metal ions and the $p$-orbitals of halide ions, corresponding to an overall $s$ symmetry with the hole angular momentum of $|1 / 2, \pm 1 / 2\rangle$. (a)

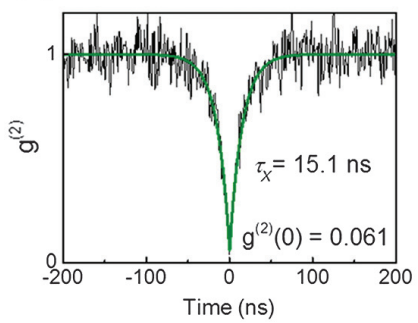

(c)

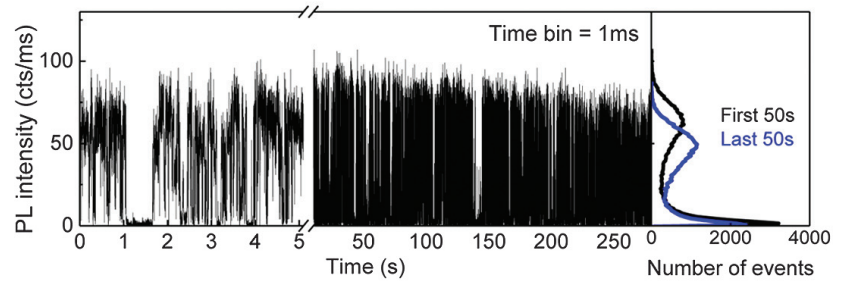

(b)

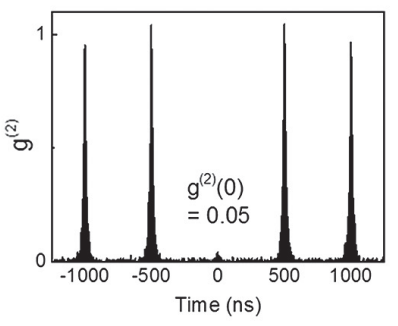

Number of events (d)

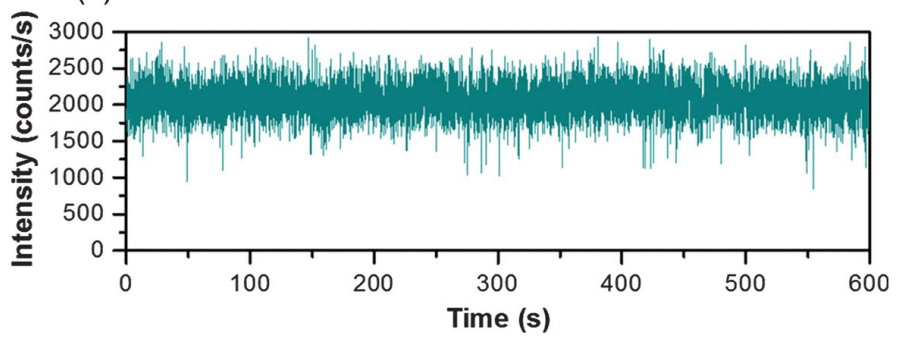

(e)

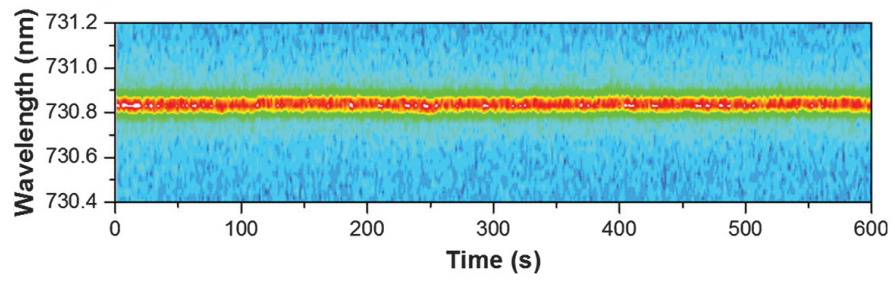

Fig. 6 Second-order autocorrelation functions measured for a single $\mathrm{CsPbl}_{3} \mathrm{NC}$ under both (a) continuous-wave and (b) pulsed laser excitations. (c) PL intensity time trace and the corresponding distribution histograms recorded for a single $\mathrm{CsPbl}_{3} \mathrm{NC}$. (d) PL intensity time trace measured for a single $\mathrm{CsPbl}_{3} \mathrm{NC}$ with suppressed fluorescence blinking. (e) Time-dependent $\mathrm{PL}$ spectral image measured for a single $\mathrm{CsPbl}_{3} \mathrm{NC}$ with suppressed spectral diffusion. (a)-(c) Reproduced with permission from Ref. 123, courtesy of ACS. (d), (e) Reproduced with permission from Ref. 124, courtesy of ACS. 
Meanwhile, the conduction band minimum is contributed by the $p$-orbitals of metal ions and, due to mixing of their wave functions with the electron spin through spin-orbit coupling, a doubly degenerate electron state is also obtained with the angular momentum of $|1 / 2, \pm 1 / 2\rangle$. For the as-formed band-edge excitons, the electron-hole exchange interaction would take further effect to result in a spin-forbidden, dark singlet state and a spinallowed, bright triplet state with the total angular momentums of 0 and 1 , respectively.

Preliminary evidence for the above exciton fine structures was provided by Rainò et al. ${ }^{127}$ in their cryogenic-temperature optical studies of single $\mathrm{CsPb}(\mathrm{Cl} / \mathrm{Br})_{3} \mathrm{NCs}$; the three-peaked PL spectrum shown in Fig. 7(a) suggested a complete splitting of the triplet bright-exciton states. Later on, it was reported by Yin et al. ${ }^{133}$ that mainly doublet PL peaks were emitted by single $\mathrm{CsPbI}_{3} \mathrm{NCs}$, while Fu et al. ${ }^{134}$ observed either doublet or triplet
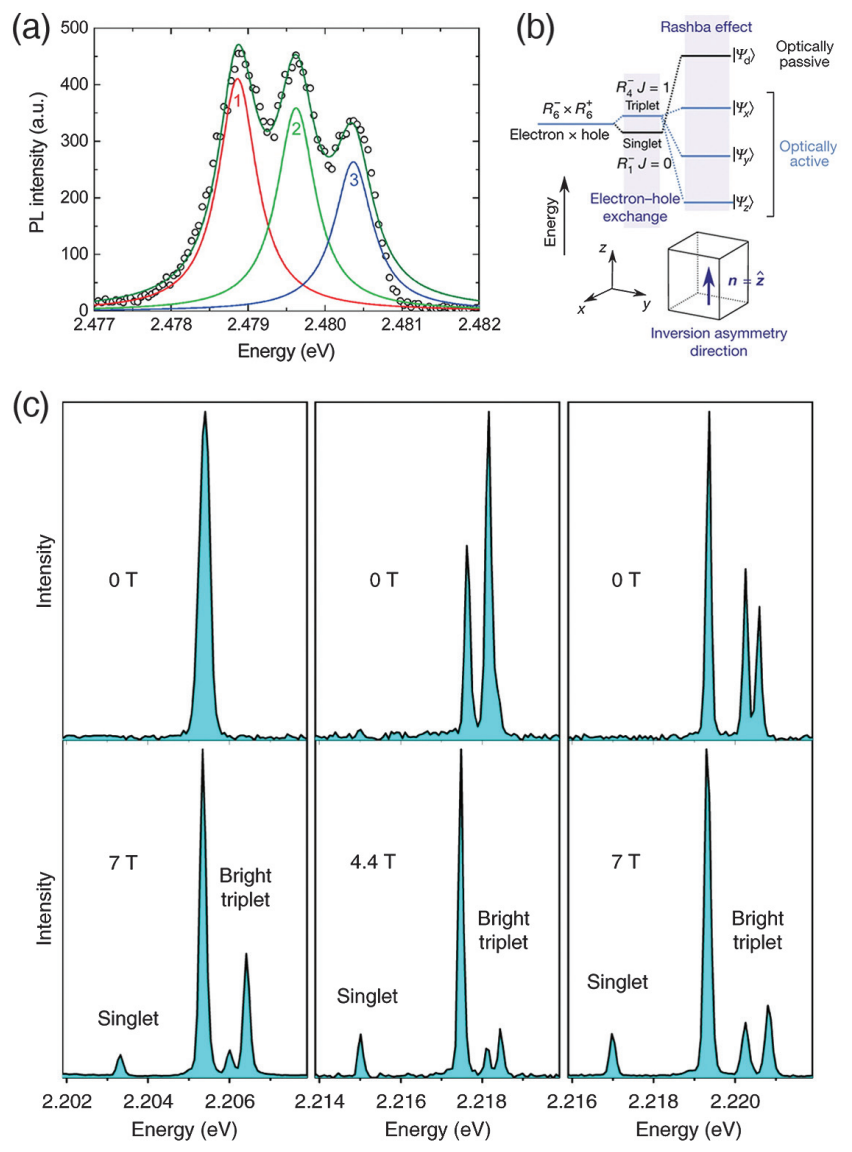

Fig. 7 (a) PL spectrum measured for a single $\mathrm{CsPb}(\mathrm{Cl} / \mathrm{Br})_{3} \mathrm{NC}$ with three emission peaks. (b) Schematic for the energy-level structures of band-edge excitons in metal-halide perovskite NCs, under the influences of both electron-hole exchange interaction and the Rashba effect. (c) One-, two-, and three-peaked PL spectra measured for three different $\mathrm{FAPbBr}_{3} \mathrm{NCs}$ at zero magnetic field (upper panels), and their corresponding four-peaked $\mathrm{PL}$ spectra measured at the magnetic fields of $7,4.4$, and $7 \mathrm{~T}$ (lower panels), respectively, all revealing the lowest-energy singlet dark-exciton peak. (a) Reproduced with permission from Ref. 127, courtesy of ACS. (b) Reproduced with permission from Ref. 132, courtesy of Macmillan Publishers Limited. (c) Reproduced with permission from Ref. 128, courtesy of Springer Nature Limited.
PL peaks from their optical studies of single $\mathrm{CsPbBr}_{3} \mathrm{NCs}$. At that time, the various types of PL peaks presented by single perovskite NCs could be largely explained by the electron-hole exchange interaction model, with the form of exciton fine-structure splittings being critically dependent on the underlying crystal phases. ${ }^{134-136}$ Specifically, the triply degenerate bright-exciton state in the cubic phase is split into a doubly degenerate state and a nondegenerate state in the tetragonal phase, the former of which would be further split into two nondegenerate states in the orthorhombic phase. While the orthorhombic phase seems to be taken by the $\mathrm{CsPbX}_{3}$ bulk and $\mathrm{NC}$ ensembles at room temperature, ${ }^{137-140}$ it was proposed by $\mathrm{Fu}$ et al. ${ }^{134}$ that the single $\mathrm{CsPbBr}_{3}$ NCs contained even in the same batch of the sample could possess different crystal phases at cryogenic temperatures. By studying single $\mathrm{CsPbI}_{3}$ NCs from the fresh and deteriorated samples, Yin et al. ${ }^{141}$ observed that they were associated with the doublet and triplet PL peaks, respectively, implying the occurrence of a crystal phase transition that resulted in a reduced crystal field to significantly modify the exciton fine structures.

From the magneto-optical measurements of single $\mathrm{CsPbBr}_{3}$ $\mathrm{NCs}$, Isarov et al. ${ }^{142}$ proposed alternatively that the exciton finestructure splittings observed at a zero magnetic field might be a direct consequence of the Rashba effect, when considering strong spin-orbit coupling in metal-halide perovskites and possible symmetry breaking due to surface or lattice imperfections. The influence of the Rashba effect on the exciton fine structures was then thoroughly studied by Becker et al., ${ }^{132}$ a major conclusion of which was that the singlet dark-exciton state should be located higher in energy than those of the triplet bright-exciton ones [Fig. 7(b)]. This intriguing energy-level alignment could be invoked to explain why the dark excitons are non-emissive and the bright excitons are highly fluorescent in common optical measurements, since their interaction channels are effectively closed in a single perovskite NC. In great contrast, the dark excitons of traditional CdSe NCs have been confirmed to occupy the lowest position in the energy-level structures, making it easy to observe their optical emission either directly or by applying a magnetic field. ${ }^{143,144}$

Very recently, Tamarat et al. ${ }^{128}$ performed magneto-optical measurements on single $\mathrm{FAPbBr}_{3} \mathrm{NCs}$ and it turned out that the dark-exciton emission could be brightened but with the lowest energy [Fig. 7(c)], which contradicted what had been proposed by Becker et al. ${ }^{132}$ in the Rashba effect model. This strongly implies that the electron-hole exchange interaction should play a dominant role in determining the exciton fine structures at least in this perovskite NC system, and the thermalmixing effect between bright and dark excitons should be extremely weak due to the requirement of two LO phonons in their spin-flip processes. ${ }^{145}$ Theoretically, it was still speculated by Sercel et al. ${ }^{146}$ that there might exist a competition between the electron-hole exchange interaction and the Rashba effect, with the latter one being more prevalent in perovskite NCs with larger dimensions or, equivalently, weaker quantum confinement. It is obvious that there are still ongoing debates as to the real origin of exciton fine structures in single perovskite NCs, which might be further influenced by the crystal phases and the shape anisotropy. ${ }^{128,135,147}$ However, the stable existence of exciton fine structures has rendered unprecedented research opportunities in coherently manipulating the exciton wave functions for novel atomic and quantum physics, as well as for practical quantum-information-processing applications. 


\subsection{Coherent Optical Properties}

Coherent optical control of the fine-structured exciton states was achieved in single epitaxial quantum dots (QDs) in $1998,{ }^{148}$ followed by the subsequent demonstration of Rabi oscillations, ${ }^{149}$ vacuum Rabi splittings, ${ }^{150}$ and Mollow triplets ${ }^{151}$ that have pushed these solid-state artificial atoms firmly into quantum information technologies. The above achievements in single epitaxial QDs rely critically on their suppressed fluorescence blinking and spectral diffusion effects, which result in stable and ultranarrow PL linewidths in the fine-structured exciton peaks. Now that the newly emerged single perovskite NCs have been equipped with quite similar optical stabilities, it is time for them to undergo intensive coherent optical investigations in which the additional benefit of emitting at visible wavelengths should make it easier for effective photon manipulations and detections.

The coherent optical studies of perovskite NCs started with the ensemble $\mathrm{CsPbBr}_{2} \mathrm{Cl}$ case in 2018 wherein Becker et al. ${ }^{152}$ utilized the transient four-wave mixing technique at $5 \mathrm{~K}$ to probe a dephasing time of $24.5 \mathrm{ps}$ for the photogenerated excitons coupled coherently with the phonon modes. Utzat et al. ${ }^{153}$ switched the coherent optical studies to single $\mathrm{CsPbBr}_{3} \mathrm{NCs}$ in 2019, with their residual spectral diffusion being compensated by the PCFS technique whose working principle and optical setup are schematically shown in Fig. 8(a). As analyzed from the PCFS data shown in Fig. 8(b), the dephasing time of $\sim 80$ ps estimated for emission-state excitons was close to the radiative lifetime of $\sim 210 \mathrm{ps}$, suggesting the possibility of generating indistinguishable single photons from a single $\mathrm{CsPbBr}_{3}$ NC. Using the Michelson-type interferometric setup plotted in Fig. 8(c), Lv et al. ${ }^{154}$ moved one step further to coherently manipulating the wave functions of absorption-state excitons in a single $\mathrm{CsPbI}_{3} \mathrm{NC}$ excited by two time-delayed laser pulses. As shown in Fig. 8(d), the two exciton wave functions thus created could interfere coherently as a function of the time delay, which was monitored by the corresponding PL intensity oscillations to estimate a dephasing time of $\sim 10$ ps for absorption-state excitons. The coherent single-photon emission and quantum interference effect reported in the above works have added two critical quantum features to the current optical studies of single perovskite NCs, and the chemical synthesis procedures and optical characterization tools should be continuously
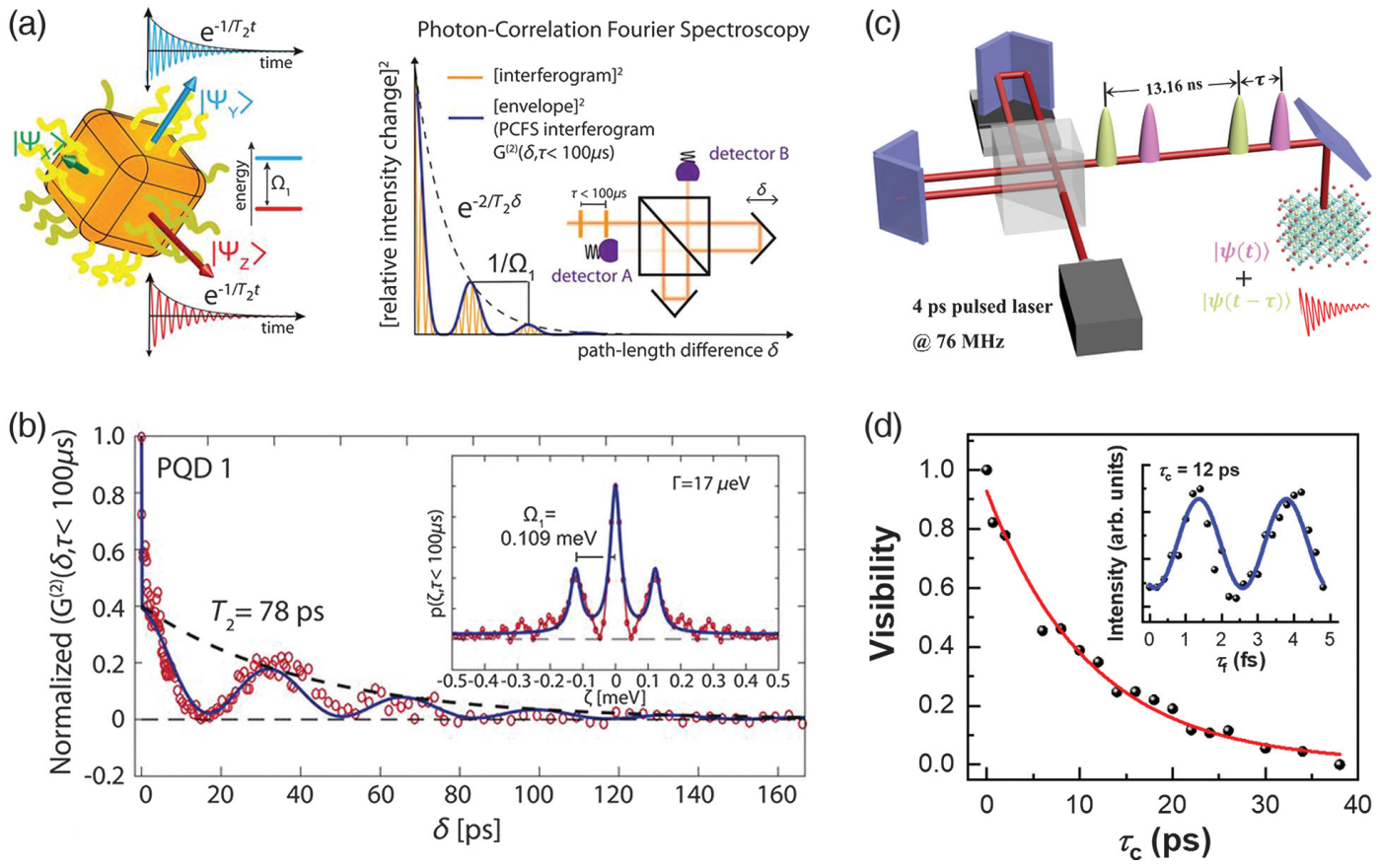

Fig. 8 (a) Schematic for the PCFS measurement of a single $\mathrm{CsPbBr}_{3} \mathrm{NC}$ with an energy separation of $\Omega_{1}$ between the two fine-structured exciton states of $\left|\Psi_{Y}\right\rangle$ and $\left|\Psi_{Z}\right\rangle$. For a short interphoton arrival time of $\tau<100 \mu \mathrm{s}$ and with the variation of the path-length difference $\delta$, PCFS measures the envelope of the interferogram squared, which is modulated at a frequency of $1 / \Omega_{1}$ with a decaying amplitude of $e^{-2 / T_{2} \delta}$. (b) For a specific single $\mathrm{CsPbBr}_{3} \mathrm{NC}$ with $\Omega_{1}=0.109 \mathrm{meV}$, an exciton dephasing time of $T_{2}=78 \mathrm{ps}$ can be fitted from the PCFS data, corresponding to a PL linewidth of $\Gamma=17 \mu \mathrm{eV}$ estimated from the Fourier-transformed spectral correlation (inset). (c) Schematic for the quantum interference measurement of a single $\mathrm{CsPbl}_{3}$ $\mathrm{NC}$, excited by two trains of picosecond laser pulses with the coarse and fine time delays of $\tau_{c}$ and $\tau_{f}$, respectively. (d) PL intensity measured at $\tau_{c}=12 \mathrm{ps}$ for a single $\mathrm{CsPbl}_{3} \mathrm{NC}$ as a function of $\tau_{f}$, showing an oscillating behavior due to quantum interference between the two exciton wave functions (inset). The oscillating amplitudes of PL intensities obtained at different $\tau_{c}$ values could be exponentially fitted to yield an exciton dephasing time of 11.12 ps. (a), (b) Reproduced with permission from Ref. 153, courtesy of the American Association for the Advancement of Science. (c), (d) Reproduced with permission from Ref. 154, courtesy of ACS. 
polished to help expedite their transition process from classical to coherent optics.

\subsection{Two-Photon Excitation}

The two-photon excitation studies of semiconductor colloidal $\mathrm{NCs}$ are aimed mainly at promoting their bioimaging and bio-labeling applications, owing to the higher spatial resolution, deeper penetration depth, and smaller sample damage as compared to those of one-photon excitation. ${ }^{155,156}$ Due to the small absorption cross section possessed by traditional colloidal NCs, most of the previous two-photon excitation studies have been performed at the ensemble level. There only exists a limited number of reports on the two-photon excitation studies of single colloidal metal-chalcogenide NCs, where their fundamental optical properties such as single-photon emission, ${ }^{157}$ spectral diffusion, ${ }^{158}$ and fluorescence blinking ${ }^{159}$ were briefly investigated.

The two-photon absorption cross sections have been estimated for ensemble perovskite NCs by means of collection efficiency, ${ }^{108,160}$ Z-scan, ${ }^{107,109,160-162}$ and $\mathrm{TA}^{163}$ measurements. The obtained values fall within the range of $\sim 10^{4}$ to $10^{6} \mathrm{GM}$, which are tremendously larger than the two-photon absorption cross sections possessed by traditional metal-chalcogenide NCs. ${ }^{156,164}$ This large absorption cross section has been utilized to realize two-photon pumped ASE and lasing actions in perovskite NC ensembles, ${ }^{106,109}$ as already detailed in a previous section of this review. In 2019, Cao et al. ${ }^{165}$ made the first two-photon excited PL measurements on single perovskite $\mathrm{NCs}$ of $\mathrm{CsPbI}_{3}$, demonstrating a better spatial resolution in the confocal scanning PL images than that from one-photon excitation [see Figs. 9(a)-9(c)]. Under both two- and one-photon excitations, quite similar optical properties were measured in the
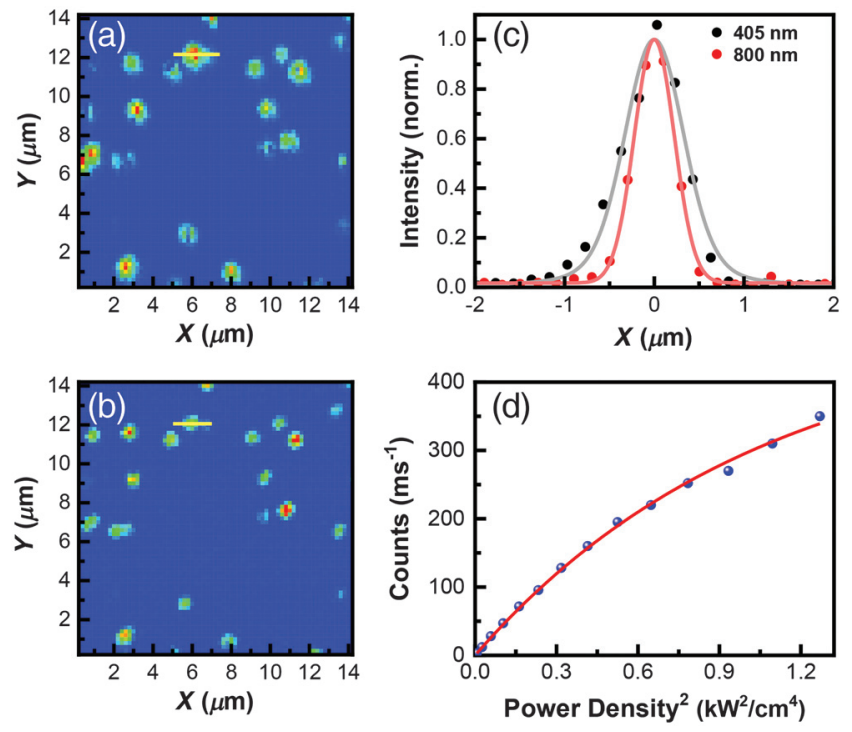

Fig. 9 Confocal scanning PL images of single $\mathrm{CsPbl}_{3} \mathrm{NCs}$ excited at (a) $405 \mathrm{~nm}$ and (b) $800 \mathrm{~nm}$, respectively. (c) PL intensity profiles drawn across the solid lines in (a) and (b), and fitted with the Gaussian distributions, respectively. (d) PL intensities of a single $\mathrm{CsPbl}_{3} \mathrm{NC}$ plotted as a function of the square of the excitation laser power density, showing the PL saturation effect. Reproduced with permission from Ref. 165, courtesy of AIP Publishing. fluorescent blinking, PL lifetime, and single-photon emission of the same single $\mathrm{CsPbI}_{3} \mathrm{NC}$, but an extra advantage of strongly reduced background fluorescence was obtained in the former case. Based on the PL saturation measurements [see Fig. 9(d)], the authors developed a new method in this report to estimate an average two-photon absorption cross section of $\sim 6.8 \times 10^{6} \mathrm{GM}$ for single $\mathrm{CsPbI}_{3} \mathrm{NCs}$, which could be extended to other ensemble and single semiconductor nanostructures influenced by multiple-exciton nonradiative Auger recombination.

In single epitaxial QDs, the two-photon excitation technique has proved to be useful in resonantly driving the biexciton states ${ }^{166}$ to realize indistinguishable polarization-entangled photon pairs. ${ }^{167,168}$ For resonant excitation of the single-exciton state for elongating the exciton dephasing time ${ }^{151}$ the complex sample designs and optical techniques are not required in the twophoton excitation approach to isolate the scattered laser light from the fluorescence signal of a single epitaxial QD. In this sense, the two-photon excited PL can go well beyond fundamental optical characterization of single perovskite NCs to serve as a powerful tool in interrogating their coherent optical properties discussed in the previous section.

\section{Conclusions and Perspective}

In this review, we summarize recent progress made on the optical studies of perovskite NCs, which can serve double purposes, for researchers to get familiar with this novel type of semiconductor nanostructure and to seek unique opportunities in fundamental physics and practical applications. The colloidal perovskite NCs can now be cheaply and facilely synthesized, with their ion compositions and structural parameters being widely tuned by versatile chemical methods. It is not surprising to see that the perovskite NCs possess many optical properties similar to those of traditional colloidal NCs, such as metal chalcogenides, ${ }^{59,169-173}$ but they additionally exhibit a rich spectrum of unique features at both the ensemble and single-particle levels. As revealed from the time-resolved PL and TA measurements, the charge carriers in perovskite NCs are featured with high transport rates in the films and can be conveniently extracted to other interfacing materials, which are both crucial for efficient operation of solar cell devices. While the above optical studies are directed to the development of commercial optoelectronic devices, the unique material and electronic properties have made it possible to observe superfluorescence from perovskite NC ensembles. This novel optical phenomenon can be potentially manipulated not only to realize classical laser operations with ultranarrow linewidths and tunable wavelengths but also to promote new-concept applications in multiplephoton quantum light sources.

Even more quantum applications can be envisioned from the optical studies of single perovskite NCs, whose defect tolerance is critical for achieving the single-photon emission behavior with suppressed PL blinking and spectral diffusion. With the subsequent revelation of stable exciton fine structures, single perovskite NCs are now standing out in the forefront of all currently available colloidal nanostructures for mimicking the discrete energy levels of natural atoms. Compared to traditional metal-chalcogenide NCs possessing a total of eight energy levels for the band-edge excitons, ${ }^{174}$ only three bright-exciton states and one dark-exciton state need to be considered in the perovskite NC case, thus greatly simplifying the theoretical explanations and experimental manipulations. In the Rashba effect theory applied to single perovskite NCs, a fascinating scenario 
is proposed to place the dark-exciton state higher in energy than those of the bright-exciton ones. However, very recent magnetooptical measurements have confirmed that the dark-exciton state is with the lowest energy at least in some types of single perovskite NCs, thus pointing to the electron-hole exchange interaction as the most likely origin for the exciton fine structures. From quantum interference measurement on one of the finestructured absorption states, the exciton dephasing time of single perovskite NCs has been successfully estimated, which marks the first step toward pushing it into the exciting regime of quantum information technologies.

As another type of important semiconductor nanostructure, single epitaxial QDs are now being widely employed within the context of quantum information technologies, starting mainly from their first quantum interference measurement in $1998,{ }^{148}$ which has since triggered observations of various other coherent optical properties, mainly including Rabi oscillations, ${ }^{149}$ vacuum Rabi splittings, ${ }^{150}$ and Mollow triplets. ${ }^{151}$ The same research route will be naturally adopted in the optical studies of single perovskite NCs, not only to reproduce the above traditional coherent optical properties but also to harvest new quantum physics dictated by their unique material and electronic properties such as the soft lattice and the large polaron effect. ${ }^{89,175}$ Meanwhile, it is possible to remove the exciton fine structures of a single perovskite NC by applying external magnetic and electric fields, so that entangled photon pairs can be generated at the visible wavelengths to compensate those emitted by single epitaxial QDs at the near-infrared spectral range.

Finally, it should be noted that, as a novel type of semiconductor nanostructure, perovskite NCs are of course facing a bunch of challenging problems that need to be solved in future explorations. The chemical stability of metal-halide perovskite NCs can be strongly influenced by the surrounding environment and even light illumination at room temperature, ${ }^{176}$ which has slowed down their large-scale commercialization in classical optoelectronic devices. The chemical routes need to be continuously polished for the synthesis of more robust perovskite NCs along with judicious controls over their sizes, which are now falling largely within the weak to intermediate regimes of quantum confinement. ${ }^{128,132,135,136,146,147}$ The chemical stability is not an obvious issue for single perovskite NCs studied at cryogenic temperatures. However, their PL linewidth is almost one order of magnitude broader than that routinely measured for single epitaxial QDs. ${ }^{177}$ This thus poses a serious concern as to whether the PL linewidth can be further reduced by the removal of residual spectral diffusion, or it is intrinsic to single perovskite $\mathrm{NCs}$ as an ultimate limit that is unsurmountable. Besides the quantum interference effect, more coherent optical properties are yet to be demonstrated from single perovskite NCs, so that they can firmly bridge the research gap between colloidal NCs and epitaxial QDs where current investigations are focused on classical optoelectronic devices and quantum information technologies, respectively.

\section{Acknowledgments}

This work was supported by the National Key R\&D Program of China (Grant Nos. 2019YFA0308700 and 2017YFA0303700), the National Natural Science Foundation of China (Grant Nos. 61974058, 11574147, and 11974164), and the PAPD of Jiangsu Higher Education Institutions.

\section{References}

1. G. Rose, De novis quibusdam fossilibus quae in montibus uraliis inveniuntur, AG Schade, Berlin (1839).

2. A. R. Chakhmouradian and P. M. Woodward, "Celebrating 175 years of perovskite research: a tribute to Roger H. Mitchell," Phys. Chem. Miner. 41, 387-391 (2014).

3. S. Bai, Z. Yuan, and F. Gao, "Colloidal metal halide perovskite nanocrystals: synthesis, characterization, and applications," J. Mater. Chem. C 4, 3898-3904 (2016).

4. C. Yang et al., "Nanocrystals of halide perovskite: synthesis, properties, and applications," J. Energy Chem. 27, 622-636 (2018).

5. L. Protesescu et al., "Nanocrystals of cesium lead halide perovskites $\left(\mathrm{CsPbX}_{3}, \mathrm{X}=\mathrm{Cl}, \mathrm{Br}\right.$, and I): novel optoelectronic materials showing bright emission with wide color gamut," Nano Lett. 15, 3692-3696 (2015).

6. H. L. Wells, "Über die cäsium- und kalium-bleihalogenide," Z. Anorg. Chem. 3, 195-210 (1893).

7. A. Kojima et al., "Organometal halide perovskites as visible-light sensitizers for photovoltaic cells," J. Am. Chem. Soc. 131, 60506051 (2009).

8. I. Chung et al., "All-solid-state dye-sensitized solar cells with high efficiency," Nature 485, 486-489 (2012).

9. H.-S. Kim et al., "Lead iodide perovskite sensitized all-solidstate submicron thin film mesoscopic solar cell with efficiency exceeding 9\%," Sci. Rep. 2, 591 (2012).

10. M. M. Lee et al., "Efficient hybrid solar cells based on mesosuperstructured organometal halide perovskites," Science 338, 643-647 (2012).

11. N.-G. Park, "Perovskite solar cells: an emerging photovoltaic technology," Mater. Today 18, 65-72 (2015).

12. J.-P. Correa-Baena et al., "Promises and challenges of perovskite solar cells," Science 358, 739-744 (2017).

13. M. L. Petrus et al., "Capturing the sun: a review of the challenges and perspectives of perovskite solar cells," Adv. Energy Mater. 7, 1700264 (2017).

14. Q. Jiang et al., "Surface passivation of perovskite film for efficient solar cells," Nat. Photonics 13, 460-466 (2019).

15. National Renewable Energy Laboratory, "Best research-cell efficiency chart," 2020, https://www.nrel.gov/pv/cell-efficiency .html.

16. Z.-K. Tan et al., "Bright light-emitting diodes based on organometal halide perovskite," Nat. Nanotechnol. 9, 687-692 (2014).

17. F. Deschler et al., "High photoluminescence efficiency and optically pumped lasing in solution-processed mixed halide perovskite semiconductors," J. Phys. Chem. Lett. 5, 1421-1426 (2014).

18. V. D'Innocenzo et al., "Tuning the light emission properties by band gap engineering in hybrid lead halide perovskite," J. Am. Chem. Soc. 136, 17730-17733 (2014).

19. S. D. Stranks and H. J. Snaith, "Metal-halide perovskites for photovoltaic and light-emitting devices," Nat. Nanotechnol. 10, 391-402 (2015).

20. J. Shamsi et al., "Metal halide perovskite nanocrystals: synthesis, post-synthesis modifications, and their optical properties," Chem. Rev. 119, 3296-3348 (2019).

21. L. C. Schmidt et al., "Nontemplate synthesis of $\mathrm{CH}_{3} \mathrm{NH}_{3} \mathrm{PbBr}_{3}$ perovskite nanoparticles," J. Am. Chem. Soc. 136, 850-853 (2014).

22. D. Zhang et al., "Solution-phase synthesis of cesium lead halide perovskite nanowires," J. Am. Chem. Soc. 137, 9230-9233 (2015).

23. Q. A. Akkerman et al., "Solution synthesis approach to colloidal cesium lead halide perovskite nanoplatelets with monolayerlevel thickness control," J. Am. Chem. Soc. 138, 1010-1016 (2016).

24. D. N. Dirin et al., "Harnessing defect-tolerance at the nanoscale: highly luminescent lead halide perovskite nanocrystals in mesoporous silica matrixes," Nano Lett. 16, 5866-5874 (2016). 
25. F. Zhang et al., "Brightly luminescent and color-tunable colloidal $\mathrm{CH}_{3} \mathrm{NH}_{3} \mathrm{PbX}_{3}(\mathrm{X}=\mathrm{Br}, \mathrm{I}, \mathrm{Cl})$ quantum dots: potential alternatives for display technology," ACS Nano 9, 4533-4542 (2015).

26. S. Sun et al., "Ligand-mediated synthesis of shape-controlled cesium lead halide perovskite nanocrystals via reprecipitation process at room temperature," ACS Nano 10, 3648-3657 (2016).

27. J. A. Sichert et al., "Quantum size effect in organometal halide perovskite nanoplatelets," Nano Lett. 15, 6521-6527 (2015).

28. D. Zhang et al., "Ultrathin colloidal cesium lead halide perovskite nanowires," J. Am. Chem. Soc. 138, 13155-13158 (2016).

29. Q. A. Akkerman et al., "Tuning the optical properties of cesium lead halide perovskite nanocrystals by anion exchange reactions," J. Am. Chem. Soc. 137, 10276-10281 (2015).

30. V. A. Hintermayr et al., "Tuning the optical properties of perovskite nanoplatelets through composition and thickness by ligandassisted exfoliation," Adv. Mater. 28, 9478-9485 (2016).

31. G. Nedelcu et al., "Fast anion-exchange in highly luminescent nanocrystals of cesium lead halide perovskites $\left(\mathrm{CsPbX}_{3}, \mathrm{X}=\right.$ Cl, Br, I)," Nano Lett. 15, 5635-5640 (2015).

32. Y. Tong et al., "Dilution-induced formation of hybrid perovskite nanoplatelets," ACS Nano 10, 10936-10944 (2016).

33. T. C. Jellicoe et al., "Synthesis and optical properties of lead-free cesium tin halide perovskite nanocrystals," J. Am. Chem. Soc. 138, 2941-2944 (2016).

34. F. Di Stasio et al., "Near-unity photoluminescence quantum yield in $\mathrm{CsPBr}_{3}$ nanocrystal solid-state films via postsynthesis treatment with lead bromide," Chem. Mater. 29, 7663-7667 (2017).

35. F. Liu et al., "Highly luminescent phase-stable $\mathrm{CsPbI}_{3}$ perovskite quantum dots achieving near $100 \%$ absolute photoluminescence quantum yield," ACS Nano 11, 10373-10383 (2017).

36. N. Mondal, A. De, and A. Samanta, "Achieving near-unity photoluminescence efficiency for blue-violet-emitting perovskite nanocrystals," ACS Energy Lett. 4, 32-39 (2018).

37. A. Swarnkar et al., "Quantum dot-induced phase stabilization of $\alpha-\mathrm{CsPbI}_{3}$ perovskite for high-efficiency photovoltaics," Science 354, 92-95 (2016).

38. Q. A. Akkerman et al., "Strongly emissive perovskite nanocrystal inks for high-voltage solar cells," Nat. Energy 2, 16194 (2016).

39. D. Zhou et al., "Cerium and ytterbium codoped halide perovskite quantum dots: a novel and efficient downconverter for improving the performance of silicon solar cells," Adv. Mater. 29, 1704149 (2017).

40. B. Li et al., "Surface passivation engineering strategy to fullyinorganic cubic $\mathrm{CsPbI}_{3}$ perovskites for high-performance solar cells," Nat. Commun. 9, 1076 (2018).

41. J. Song et al., "Quantum dot light-emitting diodes based on inorganic perovskite cesium lead halides $\left(\mathrm{CsPbX}_{3}\right)$," Adv. Mater. 27, 7162-7167 (2015).

42. $\mathrm{X}$. Li et al., " $\mathrm{CsPbX}$ quantum dots for lighting and displays: room-temperature synthesis, photoluminescence superiorities, underlying origins and white light-emitting diodes," Adv. Funct. Mater. 26, 2435-2445 (2016).

43. G. Li et al., "Highly efficient perovskite nanocrystal lightemitting diodes enabled by a universal crosslinking method," Adv. Mater. 28, 3528-3534 (2016).

44. J. Pan et al., "Highly efficient perovskite-quantum-dot lightemitting diodes by surface engineering," Adv. Mater. 28, 87188725 (2016).

45. C. Sun et al., "Efficient and stable white LEDs with silica-coated inorganic perovskite quantum dots," Adv. Mater. 28, 1008810094 (2016).

46. J. Xing et al., "High-efficiency light-emitting diodes of organometal halide perovskite amorphous nanoparticles," ACS Nano 10, 6623-6630 (2016).

47. H. C. Yoon et al., "Study of perovskite QD down-converted LEDs and six-color white LEDs for future displays with excellent color performance," ACS Appl. Mater. Interfaces 8, 18189-18200 (2016).
48. S. Yakunin et al., "Low-threshold amplified spontaneous emission and lasing from colloidal nanocrystals of caesium lead halide perovskites," Nat. Commun. 6, 8056 (2015).

49. S. Liu et al., "Random lasing actions in self-assembled perovskite nanoparticles," Opt. Eng. 55, 057102 (2016).

50. C. H. Lin et al., "Large-area lasing and multicolor perovskite quantum dot patterns," Adv. Opt. Mater. 6, 1800474 (2018)

51. P. Ramasamy et al., "All-inorganic cesium lead halide perovskite nanocrystals for photodetector applications," Chem. Commun. 52, 2067-2070 (2016).

52. J. Zhang et al., "High-performance transparent ultraviolet photodetectors based on inorganic perovskite $\mathrm{CsPbCl}_{3}$ nanocrystals," RSC Adv. 7, 36722-36727 (2017).

53. G. H. Ahmed et al., "Giant photoluminescence enhancement in $\mathrm{CsPbCl}_{3}$ perovskite nanocrystals by simultaneous dual-surface passivation," ACS Energy Lett. 3, 2301-2307 (2018).

54. D. Li et al., "Plasmonic photonic crystals induced two-order fluorescence enhancement of blue perovskite nanocrystals and its application for high-performance flexible ultraviolet photodetectors," Adv. Funct. Mater. 28, 1804429 (2018).

55. Q. Chen et al., "All-inorganic perovskite nanocrystal scintillators," Nature 561, 88-93 (2018).

56. J. H. Heo et al., "High-performance next-generation perovskite nanocrystal scintillator for nondestructive x-ray imaging," $A d v$. Mater. 30, 1801743 (2018).

57. Y. Shirasaki et al., "Emergence of colloidal quantum-dot lightemitting technologies," Nat. Photonics 7, 13-23 (2013).

58. C. R. Kagan et al., "Building devices from colloidal quantum dots," Science 353, aac5523 (2016).

59. J. M. Pietryga et al., "Spectroscopic and device aspects of nanocrystal quantum dots," Chem. Rev. 116, 10513-10622 (2016).

60. A. L. Efros and A. L. Efros, "Interband absorption of light in a semiconductor sphere," Sov. Phys. Semicond.-USSR 16, 772775 (1982)

61. A. P. Alivisatos, "Semiconductor clusters, nanocrystals, and quantum dots," Science 271, 933-937 (1996).

62. W. E. Moerner and L. Kador, "Optical detection and spectroscopy of single molecules in a solid," Phys. Rev. Lett. 62, 2535-2538 (1989).

63. L.-Q. Li and L. M. Davis, "Single photon avalanche diode for single molecule detection," Rev. Sci. Instrum. 64, 1524-1529 (1993).

64. M. Nirmal et al., "Fluorescence intermittency in single cadmium selenide nanocrystals," Nature 383, 802-804 (1996).

65. C. Galland et al., "Two types of luminescence blinking revealed by spectroelectrochemistry of single quantum dots," Nature $\mathbf{4 7 9}$, 203-207 (2011).

66. B. Lounis et al., "Photon antibunching in single $\mathrm{CdSe} / \mathrm{ZnS}$ quantum dot fluorescence," Chem. Phys. Lett. 329, 399-404 (2000).

67. H. Htoon et al., "Linearly polarized 'fine structure' of the bright exciton state in individual CdSe nanocrystal quantum dots," Phys. Rev. B 77, 035328 (2008).

68. G. W. Wen et al., "Quantum-confined stark effects in semiconductor quantum dots," Phys. Rev. B 52, 5913-5922 (1995).

69. S. A. Empedocles and M. G. Bawendi, "Quantum-confined Stark effect in single CdSe nanocrystallite quantum dots," Science $\mathbf{2 7 8}$, 2114-2117 (1997)

70. J. Even et al., "Electronic model for self-assembled hybrid organic/perovskite semiconductors: reverse band edge electronic states ordering and spin-orbit coupling," Phys. Rev. B 86, 205301 (2012).

71. W.-J. Yin, T. Shi, and Y. Yan, "Unique properties of halide perovskites as possible origins of the superior solar cell performance," Adv. Mater. 26, 4653-4658 (2014).

72. T. M. Brenner et al., "Hybrid organic-inorganic perovskites: lowcost semiconductors with intriguing charge-transport properties," Nat. Rev. Mater. 1, 15007 (2016). 
73. M. V. Kovalenko, L. Protesescu, and M. I. Bodnarchuk, "Properties and potential optoelectronic applications of lead halide perovskite nanocrystals," Science 358, 745-750 (2017).

74. S. A. Kulkarni et al., "Perovskite nanostructures: leveraging quantum effects to challenge optoelectronic limits," Mater. Today 33, 122-140 (2020).

75. L. Protesescu et al., "Dismantling the 'red wall' of colloidal perovskites: highly luminescent formamidinium and formamidinium-cesium lead iodide nanocrystals," ACS Nano 11, 31193134 (2017).

76. C. de Weerd et al., "Energy transfer between inorganic perovskite nanocrystals," J. Phys. Chem. C 120, 13310-13315 (2016).

77. H. Zhang et al., "Phase segregation due to ion migration in allinorganic mixed-halide perovskite nanocrystals," Nat. Commun. 10, 1088 (2019).

78. B. T. Diroll, H. Zhou, and R. D. Schaller, "Low-temperature absorption, photoluminescence, and lifetime of $\mathrm{CsPbX}_{3}(\mathrm{X}=\mathrm{Cl}$, Br, I) nanocrystals," Adv. Funct. Mater. 28, 1800945 (2018).

79. K. Wei et al., "Temperature-dependent excitonic photoluminescence excited by two-photon absorption in perovskite $\mathrm{CsPbBr}_{3}$ quantum dots," Opt. Lett. 41, 3821-3824 (2016).

80. J. M. Li et al., "Temperature-dependent photoluminescence of inorganic perovskite nanocrystal films," RSC Adv. 6, 7831178316 (2016).

81. J.-P. Ma et al., "Defect-triggered phase transition in cesium lead halide perovskite nanocrystals," ACS Materials Lett. 1, 185-191 (2019).

82. J. Aneesh et al., "Ultrafast exciton dynamics in colloidal $\mathrm{CsPbr}_{3}$ perovskite nanocrystals: biexciton effect and Auger recombination," J. Phys. Chem. C 121, 4734-4739 (2017).

83. N. S. Makarov et al., "Spectral and dynamical properties of single excitons, biexcitons, and trions in cesium-lead-halide perovskite quantum dots," Nano Lett. 16, 2349-2362 (2016).

84. Q. Liu et al., "Exciton relaxation dynamics in photo-excited $\mathrm{CsPbI}_{3}$ perovskite nanocrystals," Sci. Rep. 6, 29442 (2016).

85. J. A. Castaneda et al., "Efficient biexciton interaction in perovskite quantum dots under weak and strong confinement," ACS Nano 10, 8603-8609 (2016).

86. K. Wei et al., "Observation of ultrafast exciton-exciton annihilation in $\mathrm{CsPbBr}_{3}$ quantum dots," Adv. Opt. Mater. 4, 1993-1997 (2016).

87. A. Mondal et al., "Ultrafast exciton many-body interactions and hot-phonon bottleneck in colloidal cesium lead halide perovskite nanocrystals," Phys. Rev. B 98, 115418 (2018)

88. B. B. Luo et al., "Synthesis, optical properties, and exciton dynamics of organolead bromide perovskite nanocrystals," J. Phys. Chem. C 119, 26672-26682 (2015).

89. D. Rossi et al., "Light-induced activation of forbidden exciton transition in strongly confined perovskite quantum dots," ACS Nano 12, 12436-12443 (2018).

90. G. R. Yettapu et al., "Terahertz conductivity within colloidal $\mathrm{CsPbr}_{3}$ perovskite nanocrystals: remarkably high carrier mobilities and large diffusion lengths," Nano Lett. 16, 4838-4848 (2016).

91. S. D. Stranks et al., "Electron-hole diffusion lengths exceeding 1 micrometer in an organometal trihalide perovskite absorber," Science 342, 341-344 (2013).

92. G. Xing et al., "Long-range balanced electron- and hole-transport lengths in organic-inorganic $\mathrm{CH}_{3} \mathrm{NH}_{3} \mathrm{PbI}_{3}$," Science 342, 344347 (2013).

93. A. Marchioro et al., "Unravelling the mechanism of photoinduced charge transfer processes in lead iodide perovskite solar cells," Nat. Photonics 8, 250-255 (2014).

94. J. S. Manser and P. V. Kamat, "Band filling with free charge carriers in organometal halide perovskites," Nat. Photonics 8, 737-743 (2014)

95. C. Wehrenfennig et al., "High charge carrier mobilities and lifetimes in organolead trihalide perovskites," Adv. Mater. 26, 15841589 (2014).
96. G. H. Ahmed et al., "Shape-tunable charge carrier dynamics at the interfaces between perovskite nanocrystals and molecular acceptors," J. Phys. Chem. Lett. 7, 3913-3919 (2016).

97. R. Begum et al., "Engineering interfacial charge transfer in $\mathrm{Cs} \mathrm{PbBr}_{3}$ perovskite nanocrystals by heterovalent doping," $J$. Am. Chem. Soc. 139, 731-737 (2017).

98. K. Wu et al., "Ultrafast interfacial electron and hole transfer from $\mathrm{CsPbBr}_{3}$ perovskite quantum dots," J. Am. Chem. Soc. 137, 12792-12795 (2015).

99. P. Maity, J. Dana, and H. N. Ghosh, "Multiple charge transfer dynamics in colloidal $\mathrm{CsPbr}_{3}$ perovskite quantum dots sensitized molecular adsorbate," J. Phys. Chem. C 120, 18348-18354 (2016).

100. X. Luo et al., "Picosecond multi-hole transfer and microsecond charge-separated states at the perovskite nanocrystal/tetracene interface," Chem. Sci. 10, 2459-2464 (2019).

101. J. Dana et al., "Concurrent ultrafast electron- and hole-transfer dynamics in $\mathrm{CsPbBr}_{3}$ perovskite and quantum dots," ACS Omega 3, 2706-2714 (2018).

102. X. Luo et al., "Triplet energy transfer from $\mathrm{CsPbBr}_{3}$ nanocrystals enabled by quantum confinement," J. Am. Chem. Soc. 141, 4186-4190 (2019)

103. X. Luo et al., "Mechanisms of triplet energy transfer across the inorganic nanocrystal/organic molecule interface," Nat. Commun. 11, 28 (2020).

104. K. Mase et al., "Triplet sensitization by perovskite nanocrystals for photon upconversion," Chem. Commun. 53, 8261-8264 (2017).

105. Y. Wang et al., "Stable, ultralow threshold amplified spontaneous emission from $\mathrm{CsPbBr}_{3}$ nanoparticles exhibiting trion gain," Nano Lett. 18, 4976-4984 (2018).

106. J. Pan et al., "Air-stable surface-passivated perovskite quantum dots for ultra-robust, single- and two-photon-induced amplified spontaneous emission," J. Phys. Chem. Lett. 6, 5027-5033 (2015).

107. Y. Wang et al., "Nonlinear absorption and low-threshold multiphoton pumped stimulated emission from all-inorganic perovskite nanocrystals," Nano Lett. 16, 448-453 (2016).

108. G. Nagamine et al., "Two-photon absorption and two-photoninduced gain in perovskite quantum dots," J. Phys. Chem. Lett. 9, 3478-3484 (2018).

109. Y. Xu et al., "Two-photon-pumped perovskite semiconductor nanocrystal lasers," J. Am. Chem. Soc. 138, 3761-3768 (2016).

110. C. J. Wang et al., "Light emission and amplification in charged CdSe quantum dots," J. Phys. Chem. B 108, 9027-9031 (2004).

111. K. Wu et al., "Towards zero-threshold optical gain using charged semiconductor quantum dots," Nat. Nanotechnol. 12, 1140-1147 (2017).

112. Z. P. Hu et al., "Enhanced two-photon-pumped emission from in situ synthesized nonblinking $\mathrm{CsPbBr} / \mathrm{SiO}_{2}$ nanocrystals with excellent stability," Adv. Opt. Mater. 6, 1700997 (2018).

113. G. Raino et al., "Superfluorescence from lead halide perovskite quantum dot superlattices," Nature 563, 671-675 (2018)

114. C. Zhou et al., "Cooperative excitonic quantum ensemble in perovskite-assembly superlattice microcavities," Nat. Commun. 11, 329 (2020).

115. R. Bonifacio and L. A. Lugiato, "Cooperative radiation processes in 2-level systems: superfluorescence," Phys. Rev. A 11, 15071521 (1975).

116. D. Meiser and M. J. Holland, "Steady-state superradiance with alkaline-earth-metal atoms," Phys. Rev. A 81, 033847 (2010).

117. J. G. Bohnet et al., "A steady-state superradiant laser with less than one intracavity photon," Nature 484, 78-81 (2012)

118. R. G. Neuhauser et al., "Correlation between fluorescence intermittency and spectral diffusion in single semiconductor quantum dots," Phys. Rev. Lett. 85, 3301-3304 (2000).

119. Y. Chen et al., "'Giant' multishell CdSe nanocrystal quantum dots with suppressed blinking," J. Am. Chem. Soc. 130, 5026-5027 (2008) 
120. S. Hohng and T. Ha, "Near-complete suppression of quantum dot blinking in ambient conditions," J. Am. Chem. Soc. 126, 13241325 (2004).

121. H. Qin et al., "Single-dot spectroscopy of zinc-blende CdSe/ CdS core/shell nanocrystals: nonblinking and correlation with ensemble measurements," J. Am. Chem. Soc. 136, 179-187 (2014).

122. O. Chen et al., "Compact high-quality $\mathrm{CdSe}-\mathrm{CdS}$ core-shell nanocrystals with narrow emission linewidths and suppressed blinking," Nat. Mater. 12, 445-451 (2013).

123. Y. S. Park et al., "Room temperature single-photon emission from individual perovskite quantum dots," ACS Nano 9, 10386-10393 (2015).

124. F. Hu et al., "Slow Auger recombination of charged excitons in nonblinking perovskite nanocrystals without spectral diffusion," Nano Lett. 16, 6425-6430 (2016).

125. F. Hu et al., "Superior optical properties of perovskite nanocrystals as single photon emitters," ACS Nano 9, 12410-12416 (2015).

126. D. K. Sharma et al., "Stark effect and environment-induced modulation of emission in single halide perovskite nanocrystals," ACS Nano 13, 624-632 (2019).

127. G. Raino et al., "Single cesium lead halide perovskite nanocrystals at low temperature: fast single-photon emission, reduced blinking, and exciton fine structure," ACS Nano 10, 2485-2490 (2016).

128. P. Tamarat et al., "The ground exciton state of formamidinium lead bromide perovskite nanocrystals is a singlet dark state," Nat. Mater. 18, 717-724 (2019).

129. H. Huang et al., "Lead halide perovskite nanocrystals in the research spotlight: stability and defect tolerance," ACS Energy Lett. 2, 2071-2083 (2017).

130. J. Even et al., "Importance of spin-orbit coupling in hybrid organic/inorganic perovskites for photovoltaic applications," J. Phys. Chem. Lett. 4, 2999-3005 (2013).

131. J. Even, L. Pedesseau, and C. Katan, "Analysis of multivalley and multibandgap absorption and enhancement of free carriers related to exciton screening in hybrid perovskites," J. Phys. Chem. C 118, 11566-11572 (2014).

132. M. A. Becker et al., "Bright triplet excitons in caesium lead halide perovskites," Nature 553, 189-193 (2018).

133. C. Yin et al., "Bright-exciton fine-structure splittings in single perovskite nanocrystals," Phys. Rev. Lett. 119, 026401 (2017).

134. M. Fu et al., "Neutral and charged exciton fine structure in single lead halide perovskite nanocrystals revealed by magneto-optical spectroscopy," Nano Lett. 17, 2895-2901 (2017).

135. M. O. Nestoklon et al., "Optical orientation and alignment of excitons in ensembles of inorganic perovskite nanocrystals," Phys. Rev. B 97, 235304 (2018).

136. R. Ben Aich et al., "Bright-exciton splittings in inorganic cesium lead halide perovskite nanocrystals," Phys. Rev. Appl. 11, 034042 (2019).

137. C. C. Stoumpos et al., "Crystal growth of the perovskite semiconductor $\mathrm{CsPbBr}_{3}$ : a new material for high-energy radiation detection," Cryst. Growth Des. 13, 2722-2727 (2013).

138. P. Cottinghama and R. L. Brutchey, "On the crystal structure of colloidally prepared $\mathrm{CsPbBr}_{3}$ quantum dots," Chem. Commun. 52, 5246-5249 (2016).

139. F. Bertolotti et al., "Coherent nanotwins and dynamic disorder in cesium lead halide perovskite nanocrystals," ACS Nano 11, 3819-3831 (2017)

140. Q. Zhao et al., "Size-dependent lattice structure and confinement properties in $\mathrm{CsPbI}_{3}$ perovskite nanocrystals: negative surface energy for stabilization," ACS Energy Lett. 5, 238-247 (2020).

141. C. Yin et al., "Transition from doublet to triplet excitons in single perovskite nanocrystals," J. Phys. Chem. Lett. 11, 5750-5755 (2020).
142. M. Isarov et al., "Rashba effect in a single colloidal $\mathrm{CsPbBr}_{3}$ perovskite nanocrystal detected by magneto-optical measurements," Nano Lett. 17, 5020-5026 (2017).

143. L. Biadala et al., "Direct observation of the two lowest exciton zero-phonon lines in single CdSe/ZnS nanocrystals," Phys. Rev. Lett. 103, 037404 (2009).

144. L. Biadala et al., "Band-edge exciton fine structure of single $\mathrm{CdSe} / \mathrm{ZnS}$ nanocrystals in external magnetic fields," Phys. Rev. Lett. 105, 157402 (2010).

145. M. Fu et al., "Unraveling exciton-phonon coupling in individual $\mathrm{FAPbI}_{3}$ nanocrystals emitting near-infrared single photons," Nat. Commun. 9, 3318 (2018).

146. P. C. Sercel et al., "Exciton fine structure in perovskite nanocrystals," Nano Lett. 19, 4068-4077 (2019).

147. P. C. Sercel et al., "Quasicubic model for metal halide perovskite nanocrystals," J. Chem. Phys. 151, 234106 (2019).

148. N. H. Bonadeo et al., "Coherent optical control of the quantum state of a single quantum dot," Science 282, 1473-1476 (1998).

149. T. H. Stievater et al., "Rabi oscillations of excitons in single quantum dots," Phys. Rev. Lett. 87, 133603 (2001).

150. T. Yoshie et al., "Vacuum Rabi splitting with a single quantum dot in a photonic crystal nanocavity," Nature 432, 200-203 (2004).

151. E. B. Flagg et al., "Resonantly driven coherent oscillations in a solid-state quantum emitter," Nat. Phys. 5, 203-207 (2009).

152. M. A. Becker et al., "Long exciton dephasing time and coherent phonon coupling in $\mathrm{CsPbBr}_{2} \mathrm{Cl}$ perovskite nanocrystals," Nano Lett. 18, 7546-7551 (2018).

153. H. Utzat et al., "Coherent single-photon emission from colloidal lead halide perovskite quantum dots," Science 363, 1068-1072 (2019).

154. Y. Lv et al., "Quantum interference in a single perovskite nanocrystal," Nano Lett. 19, 4442-4447 (2019).

155. H. E. Grecco et al., "Ensemble and single particle photophysical properties (two-photon excitation, anisotropy, FRET, lifetime, spectral conversion) of commercial quantum dots in solution and in live cells," Microsc. Res. Tech. 65, 169-179 (2004).

156. R. Zhang et al., "Two-photon 3D FIONA of individual quantum dots in an aqueous environment," Nano Lett. 11, 4074-4078 (2011).

157. M. D. Wissert et al., "Quantum dots as single-photon sources: antibunching via two-photon excitation," Phys. Rev. B 83, 113304 (2011)

158. S. A. Blanton, M. A. Hines, and P. Guyot-Sionnest, "Photoluminescence wandering in single CdSe nanocrystals," Appl. Phys. Lett. 69, 3905-3907 (1996).

159. K. T. Early and D. J. Nesbitt, "Ultrafast laser studies of twophoton excited fluorescence intermittency in single CdSe/ZnS quantum dots," Nano Lett. 15, 7781-7787 (2015).

160. A. Pramanik et al., "Several orders-of-magnitude enhancement of multiphoton absorption property for $\mathrm{CsPbX}_{3}$ perovskite quantum dots by manipulating halide stoichiometry," J. Phys. Chem. C 123, 5150-5156 (2019).

161. T. C. He et al., "Strong two-photon absorption of Mn-doped $\mathrm{CsPbCl}_{3}$ perovskite nanocrystals," Appl. Phys. Lett. 111, 211105 (2017).

162. Q. J. Han et al., "Two-photon absorption and upconversion luminescence of colloidal $\mathrm{CsPbX}_{3}$ quantum dots," Opt. Mater. 75, 880-886 (2018).

163. J. Chen et al., "Size- and wavelength-dependent two-photon absorption cross-section of $\mathrm{Cs} \mathrm{PbBr}_{3}$ perovskite quantum dots," J. Phys. Chem. Lett. 8, 2316-2321 (2017).

164. N. S. Makarov et al., "Two-photon absorption in CdSe colloidal quantum dots compared to organic molecules," ACS Nano $\mathbf{8}$, 12572-12586 (2014).

165. Z. Cao et al., "Two-photon excited photoluminescence of single perovskite nanocrystals," J. Chem. Phys. 151, 154201 (2019). 
166. H. Jayakumar et al., "Deterministic photon pairs and coherent optical control of a single quantum dot," Phys. Rev. Lett. 110, 135505 (2013).

167. M. Mueller et al., "On-demand generation of indistinguishable polarization-entangled photon pairs," Nat. Photonics 8, 224-228 (2014).

168. D. Huber et al., "Strain-tunable GaAs quantum dot: a nearly dephasing-free source of entangled photon pairs on demand," Phys. Rev. Lett. 121, 033902 (2018).

169. D. E. Gómez, M. Califano, and P. Mulvaney, "Optical properties of single semiconductor nanocrystals," Phys. Chem. Chem. Phys. 8, 4989-5011 (2006).

170. N. Gaponik et al., "Progress in the light emission of colloidal semiconductor nanocrystals," Small 6, 1364-1378 (2010).

171. J. Y. Kim et al., "Colloidal quantum dot materials and devices: a quarter-century of advances," Adv. Mater. 25, 4986-5010 (2013).

172. M. J. Fernée, P. Tamarat, and B. Lounis, "Spectroscopy of single nanocrystals," Chem. Soc. Rev. 43, 1311-1337 (2014).

173. M. V. Kovalenko et al., "Prospects of nanoscience with nanocrystals," ACS Nano 9, 1012-1057 (2015).
174. M. Nirmal et al., "Observation of the "dark exciton" in CdSe quantum dots," Phys. Rev. Lett. 75, 3728-3731 (1995).

175. X.-Y. Zhu and V. Podzorov, "Charge carriers in hybrid organicinorganic lead halide perovskites might be protected as large polarons," J. Phys. Chem. Lett. 6, 4758-4761 (2015).

176. F. Yan and H. V. Demir, "LEDs using halide perovskite nanocrystal emitters," Nanoscale 11, 11402 (2019).

177. D. Gammon et al., "Homogeneous linewidths in the optical spectrum of a single gallium arsenide quantum dot," Science 273, 87-90 (1996).

Xiaoyong Wang received his bachelor and master degrees of optical engineering from Tianjin University, China and his $\mathrm{PhD}$ of physics from University of Arkansas at Fayetteville, USA. He took postdoctoral positions in the physics department of the University of Texas at Austin and then in the chemistry department of the University of Rochester, USA. Currently, he is a full professor in the school of physics at Nanjing University, China.

Biographies of the other authors are not available. 